\title{
Synaptic Homeostasis Is Consolidated by the Cell Fate Gene gooseberry, a Drosophila pax3/7 Homolog
}

\author{
Bruno Marie, Edward Pym, Sharon Bergquist, and Graeme W. Davis \\ Department of Biochemistry and Biophysics, University of California San Francisco, San Francisco, California 94158-2822
}

In a large-scale screening effort, we identified the gene gooseberry $(g s b)$ as being necessary for synaptic homeostasis at the Drosophila neuromuscular junction. The $g s b$ gene encodes a pair-rule transcription factor that participates in embryonic neuronal cell fate specification. Here, we define a new postembryonic role for gooseberry. We show that $g s b$ becomes widely expressed in the postembryonic CNS, including within mature motoneurons. Loss of $g s b$ does not alter neuromuscular growth, morphology, or the distribution of essential synaptic proteins. However, $g s b$ function is required postembryonically for the sustained expression of synaptic homeostasis. In GluRIIA mutant animals, miniature EPSP (mEPSP) amplitudes are significantly decreased, and there is a compensatory homeostatic increase in presynaptic release that restores normal muscle excitation. Loss of $g s b$ significantly impairs the homeostatic increase in presynaptic release in the GluRIIA mutant. Interestingly, $g s b$ is not required for the rapid induction of synaptic homeostasis. Furthermore, $g s b$ seems to be specifically involved in the mechanisms responsible for a homeostatic increase in presynaptic release, since it is not required for the homeostatic decrease in presynaptic release observed following an increase in mEPSP amplitude. Finally, Gsb has been shown to antagonize Wingless signaling during embryonic fate specification, and we present initial evidence that this activity is conserved during synaptic homeostasis. Thus, we have identified a gene $(g s b)$ that distinguishes between rapid induction versus sustained expression of synaptic homeostasis and distinguishes between the mechanisms responsible for homeostatic increase versus decrease in synaptic vesicle release.

\section{Introduction}

Throughout the nervous system there is evidence that homeostatic signaling systems can regulate neuronal excitability through modulation of synaptic transmission or ion channel abundance (Davis, 2006; Marder and Goaillard, 2006; Turrigiano, 2008). However, the molecular mechanisms responsible for the homeostatic regulation of neural function remain poorly understood. It has also been hypothesized that defective homeostatic signaling could contribute to the cause or progression of neurological disease (Bernard et al., 2004; Houweling et al., 2005; Davis, 2006; Jakubs et al., 2006). However, molecular characterization of homeostatic signaling will be necessary before clear links to neurological disease can be established.

Homeostatic signaling systems modulate synaptic transmission at the neuromuscular junction (NMJ) of organisms ranging from Drosophila to human (for review, see Davis, 2006). In Drosophila, manipulations that decrease the sensitivity of postsynaptic glutamate receptors (GluR), such as genetically deleting or altering the nonessential GluR subunit GluRIIA (Petersen et al.,

Received Nov. 4, 2009; revised April 22, 2010; accepted April 30, 2010.

This work was supported by National Institutes of Health Grant NS39313 (to G.W.D.). B.M. was supported in part by Research Centers in Ministry Institutions Grant G12 RR03051. We thank Dr. Jonathan Blagburn and Catherine Massaro for help with confocal microscopy and members of the Davis laboratory, including C. Andrew Frank, Benjamin Eaton, and Jan Pielage, for collaboration, discussion, and reading prior versions of this manuscript. We thank Dr. Markus Noll for providing UAS-gsb flies.

Correspondence should be addressed to Graeme W. Davis, University of California San Francisco, 1550 4th Street, Rock Hall, 4th Floor North, San Francisco, CA 94158-2822. E-mail: Graeme.davis@ucsf.edu.

B. Marie's present address: Institute of Neurobiology, Department of Anatomy and Neurobiology, Medical Sciences Campus, University of Puerto Rico, San Juan, Puerto Rico.

DOI:10.1523/JNEUROSCI.5467-09.2010

Copyright $\odot 2010$ the authors $\quad 0270-6474 / 10 / 308071-12 \$ 15.00 / 0$
1997; Davis et al., 1998) or applying sub-blocking concentrations of the GluR-specific toxin philanthotoxin (PhTx) (Frank et al., 2006), cause a homeostatic increase in presynaptic neurotransmitter release that precisely counteracts the decrease in postsynaptic receptor sensitivity to achieve normal neuromuscular depolarization. This is a process that is termed synaptic homeostasis (Davis and Goodman, 1998; Davis, 2006). The induction of synaptic homeostasis at the Drosophila NMJ is rapid (minutes), independent of new protein synthesis, and requires the full functionality of presynaptic $\mathrm{Ca}_{\mathrm{V}} 2.1$ calcium channels (Frank et al., 2006). Although the induction of synaptic homeostasis at this NMJ is transcription independent, a transcription-dependent mechanism involving bone morphogenic protein (BMP) signaling is required within the presynaptic motoneuron to confer competence for expression of synaptic homeostasis (Goold and Davis, 2007).

Here, we demonstrate that the transcription factor gooseberry is essential for the sustained expression of synaptic homeostasis at the Drosophila NMJ. Gooseberry was identified in a large-scale screen for genes involved in synaptic homeostasis. Gooseberry is the Drosophila homolog of vertebrate pax3 and pax7, which are involved in neuronal cell fate determination. In Drosophila, gooseberry functions as part of a combinatorial transcription factor code that specifies neuronal cell fate in the embryonic CNS ( $\mathrm{Li}$ and Noll, 1993; Skeath et al., 1995; Duman-Scheel et al., 1997; Bhat et al., 2000; Deshpande et al. 2001). Gooseberry function has never before been examined in the postembryonic nervous system. Here, we show that gooseberry is widely expressed in the postembryonic nervous system and is necessary in postmitotic 
neurons for the sustained expression of a homeostatic increase in presynaptic neurotransmitter release. These data identify a new gene involved in synaptic homeostasis and genetically separate the mechanisms responsible for the rapid induction versus the sustained expression of synaptic homeostasis. These data also genetically separate the homeostatic mechanisms responsible for a homeostatic enhancement versus a homeostatic decrease in synaptic efficacy. Finally, our data define a new postembryonic function for a class of genes previously studied only in the context of embryonic cell fate determination.

\section{Materials and Methods}

Fly genetics. The sense (with XbaI restriction site GGGCGGGTTCTAGAGCAACTCCCACTACCAGTG) and antisense (with XbaI restriction site GGGCGGGTTCTAGATATGGATAGGTTGGTGCAGTG) oligonucleotides were used as primers in a PCR on Drosophila genomic DNA to amplify part (491 bp) of a $g s b$ exon. Standard procedures were followed to insert this DNA fragment as two inverted repeats in the UAScontaining vector pWIZ (Lee and Carthew, 2003), which allows the production of hairpin RNA leading to the production of doublestranded RNA. Transgenic flies were generated by standard methods. Homozygous viable and fertile insertions on the second and third chromosome were selected. When used with the GAL4/UAS expression system (Brand and Perrimon, 1993), it allows for the conditional expression of RNA interference (RNAi). The insertions are referred to as UAS-gsb$R N A i$ in this manuscript. In our experiments, we used the elav ${ }^{C 155}$-Gal4 line to drive expression in postmitotic neurons. For simplification,

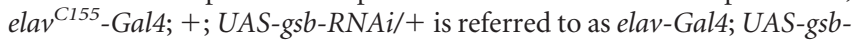
RNAi. elav ${ }^{C 155}$-Gal4; +; UAS-gsb-RNAi/UAS-gsb-RNAi is referred to as elav-Gal4; UAS-gsb-RNAi ${ }^{2 \times}$. elav ${ }^{C 155}$-Gal4;UAS-gsb-RNAi/+;UAS-gsbRNAi/UAS-gsb-RNAi is referred as elav-Gal4; UAS-gsb-RNAi ${ }^{3 \times}$. The stocks containing the $g s b^{01155}$ and $W g^{I-12}$ alleles were obtained from the Bloomington Stock Center (Indiana University, Bloomington, IN). The $g s b^{01155}$ and $W g^{I-12}$ alleles were recombined on the chromosome containing the GluRIIA sp16 allele following standard methods. The lines investigated in this manuscript are $g s b^{01155} /+$ and GluRIIA sp16, gs $b^{01155} /$ GluRIIA $^{\text {sp16 }},+$. We also examined GluRIIA ${ }^{\text {sp16 }}, \mathrm{Wg}^{I-12} /$ GluRIIA $^{\text {sp16 }},+$ and GluRIIA $A^{s p 16},+, g s b^{01155} / G l u R I I A^{s p 16}, W g^{I-12},+$. The flies containing the $W g^{I-12}$ allele were raised at restrictive temperature $\left(25-27^{\circ} \mathrm{C}\right) . U A S-$ $G s b$ flies were a gift from Markus Noll (University of Zurich, Zurich, Switzerland). The stock containing the $B x^{M S 1094}$ Gal4 enhancer trap (expression in wing imaginal discs) was obtained from the Bloomington Stock Center. We refer to this allele as MS1094-Gal4.

Immunohistochemistry. Synaptic bouton numbers were imaged and quantified as described previously (Pielage et al., 2006). The mouse monoclonal anti-Synapsin antibody (Klagges, et al., 1996) was used at 1:20 dilution. FITC-labeled anti-mouse secondary antibody (Jackson ImmunoResearch Laboratories) was used at a dilution of 1:200. The number of boutons per NMJ revealed by the Synapsin immunoreactivity was quantified at the synapse on muscle $6 / 7$ on segment A3, including 7-12 NMJs per genotype. To quantify synaptic protein abundance at the NMJ, the mouse monoclonal anti-Synapsin antibody (Klagges et al., 1996) was used at 1:20 dilution, the mouse monoclonal anti-Bruchpilot (nc82) (Wucherpfennig et al., 2003) at 1:50 dilution, the polyclonal antibody against Dlg at 1:5000 dilution (Budnik et al., 1996), and the polyclonal antibody against Dynamin at 1:200 dilution (Roos and Kelly, 1998). Secondary antibodies were used at a concentration of $1: 200$. When fluorescence intensities of different mutants and wild-type (wt) genotypes were compared, the larval fillets were processed in the same tube, treated identically, and imaged using identical settings. Imaging was performed using a Zeiss LSM5 Pascal confocal microscope. Individual entire muscle 4 NMJ synapses were optically sectioned $(0.2 \mu \mathrm{m}$; series of $12-18$ sections per synapse). A two-dimensional (2D) projection was generated that projected maximal pixel intensity. The average fluorescence of the $2 \mathrm{D}$ projection was calculated over the entire synaptic area. Software analysis was driven by ImageJ software (http://rsbweb.nih.gov/ij/). The fluorescence intensity values we present represent the difference between the synaptic intensity and muscle intensity $(\Delta \mathrm{F})$ over the intensity of the muscle (F) normalized to wild-type values. Third instar larval CNSs were dissected in HL3 saline, fixed with $4 \%$ paraformaldehyde (PFA) fixative for $15 \mathrm{~min}$, washed in PBS, and permeabilized in $0.1 \%$ Triton X-100 in PBS. A polyclonal rabbit anti $\beta$-galactosidase antibody (Invitrogen) was used at 1:5000. TRITC-labeled anti-rabbit secondary antibody (Jackson ImmunoResearch Laboratories) was used at a dilution of 1:200. Imaging was made using a Zeiss LSM5 Pascal confocal microscope.

Electrophysiological recordings. Third instar larvae were selected and dissected according to previously published techniques (Davis et al., 1998; Marie et al., 2004). Whole-muscle recordings were performed on muscle 6 in abdominal segment A3 using sharp microelectrodes (12-16 $\mathrm{M} \Omega$ ). Recordings were selected for analysis only with resting membrane potentials more hyperpolarized than $-60 \mathrm{mV}$ and with input resistances $>5$ $\mathrm{M} \Omega$. The average miniature EPSP (mEPSP) amplitude was quantified by measuring the amplitude of 100-200 individual, sequential spontaneous mEPSP events per NMJ. The average per-NMJ mEPSP amplitudes were then averaged for each genotype. Measurement of mEPSP amplitudes was semiautomated (Synaptosoft). The average superthreshold-evoked EPSP amplitude was calculated for each synapse, ensuring that both motor axons innervating muscle 6 in segment $\mathrm{A} 3$ were recruited. Quantal content (QC) was calculated as the average of EPSP amplitude divided by the average mEPSP amplitude. Quantal content was determined for each synapse and then averaged across synapses to generate the average quantal content for each genotype. Data for steady-state synaptic transmission were acquired in HL3 saline $\left(0.3 \mathrm{mM} \mathrm{Ca}^{2+} ; 10 \mathrm{mM} \mathrm{Mg}^{2+}\right)$. For experiments examining synaptic depression during sustained nerve stimulation, synapses were recorded in HL3 saline $\left(2 \mathrm{~mm} \mathrm{Ca}^{2+} ; 20 \mathrm{mM} \mathrm{Mg}^{2+}\right)$.

Recordings of central neurons were performed in wandering third instar larvae as described previously (Rohrbough and Broadie, 2002). Whole-cell recordings were achieved using thick-walled borosilicate glass electrodes (GC100F-10; Harvard Apparatus) fire polished to resistances of between 5 and $10 \mathrm{M} \Omega$. Cells were identified based on position within the ventral nerve cord, and absolute identification was determined after recording by labeling with sulfur rhodamine $(0.3 \%$; Invitrogen), which was included in the patch saline. Recordings were made using an Axopatch-200B amplifier and Digidata 1322A controlled by pClamp 9 (Molecular Devices). Only cells with an input resistance $>1$ $\mathrm{G} \Omega$ were accepted for analysis. Traces were filtered online at $5 \mathrm{kHz}$ and sampled at $10 \mathrm{kHz}$. Membrane excitability was determined using injection of depolarizing current $(10-100 \mathrm{pA} / 500 \mathrm{~ms})$ from a resting membrane potential (RMP) of $-60 \mathrm{mV}$. RMPs were maintained at $-60 \mathrm{mV}$ by injection of a small amount of hyperpolarizing current. Action potential dynamics were analyzed using the Mini Analysis program (Synaptosoft). External saline for dissection and current-clamp analysis of excitability consisted of the following (in $\mathrm{mm}$ ): $135 \mathrm{NaCl}, 5 \mathrm{KCl}$, $4 \mathrm{MgCl}_{2} \cdot 6 \mathrm{H}_{2} \mathrm{O}, 2 \mathrm{CaCl}_{2} \cdot 2 \mathrm{H}_{2} \mathrm{O}, 5 \mathrm{~N}$-Tris[hydroxymethyl]methyl-2aminoethanesulfonic acid, and 36 sucrose, $\mathrm{pH}$ 7.15. Internal patch solution consisted of (in $\mathrm{mM}$ ): $140 \mathrm{~K}^{+}$methylsulfonate $\left(\mathrm{KCH}_{3} \mathrm{SO}_{3}\right), 2$ $\mathrm{MgCl}_{2} \cdot 6 \mathrm{H}_{2} \mathrm{O}, 2$ EGTA, $5 \mathrm{KCl}$, and 20 HEPES, pH 7.4.

Quantitative reverse transcription PCR. Primer-probes specific for realtime PCR detection of Gsb and ribosomal protein L32 (RpL32) were designed and developed by Applied Biosystems. The CNS was removed from 25 third instar larvae per sample (3-6 samples/genotype). Total RNA was isolated from each sample using the standard TRIzol (Invitrogen) protocol. DNase digestion removed potential DNA contamination (RQ1 RNase-free DNase; Promega). Reverse transcription (RT) was performed (TaqMan reverse transcription reagents; Applied Biosystems) using random hexamers and $1 \mu \mathrm{g}$ of total RNA. A no RT control was performed for each sample. Purified cDNA was used as a template in 30 $\mu l$ of PCR (TaqMan Universal PCR Master Mix, no AmpErase uracil $N$-gylcosylase, Applied Biosystems). This $30 \mu \mathrm{l}$ reaction was divided into three $10 \mu \mathrm{l}$ triplicates. In addition, one $10 \mu \mathrm{l}$ no RT reaction was used for each sample. ABI Prism 7900 was used for all PCRs. Cycle threshold $\left(C_{T}\right)$ was determined by automated threshold analysis using SDS2.3 software according to the manufacturer's instructions (Applied Biosystems). Comparative Gsb levels (between wt and mutant animals) were determined using the $\Delta \Delta \mathrm{C}_{\mathrm{T}}$ method (Applied Biosystems User Bulletin No. 2). To determine whether the two amplification reactions have the same PCR efficiency, $\Delta C_{T}\left(C_{T}\right.$ of experimental gene $-C_{T}$ of reference gene $)$ 
A

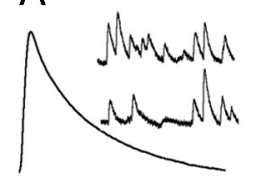
wild type

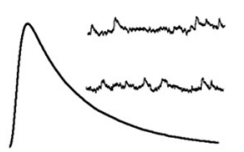
GIURIIA $1 \mathrm{mv}$
$5 \mathrm{mv}$

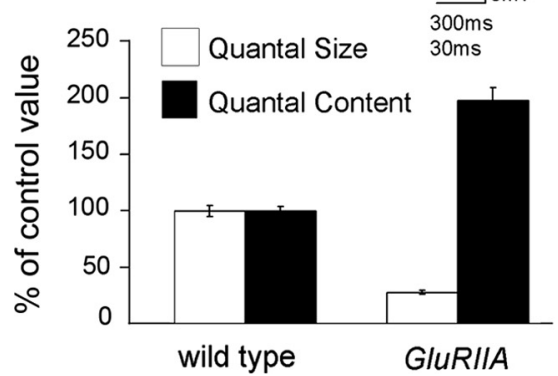

$\mathrm{B}$

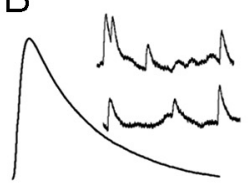
$g s b /+$

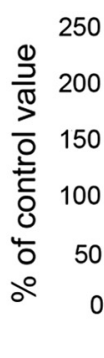

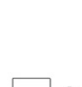

\section{.}

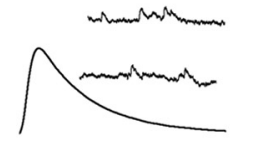

GluRIIA;gsb/+ $1 \mathrm{mv}$ $5 \mathrm{mv}$ $300 \mathrm{~ms}$ $30 \mathrm{~ms}$ Quantal Content

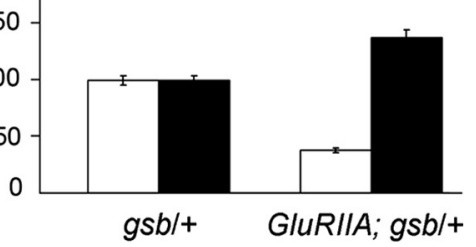

Figure 1. Disruption of synaptic homeostasis in heterozygous gsb mutant animals. A, Top, Representative traces of wild type and GluRIII ${ }^{\text {Sp16 }}$. Bottom, Quantification of average mEPSP amplitude (open bars) and quantal content (filled bars). Values are normalized to wild-type control. GluRIIA ${ }^{5 p 16}$ mutants have decreased mEPSP amplitudes and a homeostatic increase in quantal content $(p<0.001)$. B, Top: Representative traces recorded from $g \mathrm{sb}^{01155} /+$ and GluRllA ${ }^{5 p 16} ; g \mathrm{sb}^{01155} /+$. Note that there is no difference between comparing wild-type mEPSP amplitude $(0.94 \pm 0.04 \mathrm{mV} ; n=25)$ and quantal content $(41.8 \pm 1.8 ; n=25)$ and $g s b^{01155} /+\mathrm{mEPSPs}(0.88 \pm 0.04$ $\mathrm{mV} ; n=23)$ and quantal content (45.3 $\pm 1.9 ; n=23)(p=0.18)$. Bottom, Quantal content (filled bar) and mEPSP amplitude (open bar) are quantified. Quantification shows that the homeostatic increase in quantal content is significantly reduced in GluRIIA $A^{5 p 16} ; \mathrm{gs} b^{01155} /+$ animals. Although GluRIIA ${ }^{\mathrm{sp} 16}$ animals ( $\mathrm{mEPSP}=0.34 \pm 0.02 \mathrm{mV} ; \mathrm{EPSP}=27.8 \pm 1.8 \mathrm{mV}$; quantal content $=82.5 \pm 5.1 ; n=16$ ) show a considerable homeostatic increase in $Q C\left(98 \pm 12 \%\right.$ increase beyond control value; $\left.p=4.110^{-7}\right)$, the GluRIII ${ }^{\text {sp16 }}$, gsb $b^{01155}$ / GluRIIA ${ }^{\text {sp16 }},+$ animals show greatly reduced synaptic transmission ( $\mathrm{mEPSP}=0.33 \pm 0.02 \mathrm{mV}$; EPSP $=20.8 \pm 1.6 \mathrm{mV}$; quantal content $=62.3 \pm 1.3 ; n=13$ ) due to compromised homeostasis compensation. There is significantly less homeostatic compensation (only a $37 \pm 7 \%$ increase; $p=0.002$ ). Error bars represent average \pm SEM.

values are determined across the serial dilutions and plotted against the $\log$ of the cDNA dilution. Briefly, the $\Delta \Delta \mathrm{C}_{\mathrm{T}}$ method is performed as follows: (1), $\Delta \mathrm{C}_{\mathrm{T}}$ values are determined as explained above; (2) experimental animal (mutants) $\Delta \mathrm{C}_{\mathrm{T}}$ values were subtracted from control animal (wt) $\Delta \mathrm{C}_{\mathrm{T}}$ values to give the $\Delta \Delta \mathrm{C}_{\mathrm{T}}$; (3) using the equation $2^{\wedge}\left(-\Delta \Delta \mathrm{C}_{\mathrm{T}}\right) \times 100$, the percentage expression of each gene in experimental animals compared with control animals was calculated. Each experimental animal sample was compared with each wild-type sample (Applied Biosystems User Bulletin No. 2).

\section{Results}

The gooseberry gene was identified in a screen for genes that disrupt synaptic homeostasis when mutated or knocked down (Frank et al., 2009). gsb encodes a transcription factor homologous to mammalian pax3 and pax7, containing both a homeodomain and a paired domain. Null mutations in $g s b$ are embryonic lethal because of severe defects in neuroectoderm patterning. However, heterozygous null mutations are adult viable and show no evidence of altered cell fate in the neuromuscular system. In heterozygous $g s b^{01155} /+$ mutants, for example, motoneurons navigate into the periphery and establish the correct stereotyped pattern of synaptic connections with identified muscle cells (data not shown). In addition, staining the nervous system with antibodies that label subpopulations of central neurons shows no evidence of perturbed neuronal cell fate (data not shown). These data agree with previous genetic studies of cell fate determination (Gutjahr et al., 1993; Li and Noll, 1993; Skeath et al., 1995; Duman-Scheel et al., 1997).

\section{Synaptic homeostasis is inhibited by a heterozygous gsb mutation}

Glutamate receptors at the Drosophila NMJ include two nonessential subunits, GluRIIA and GluRIIB (Petersen et al., 1997; DiAntonio et al., 1999). Null mutations in the GluRIIA gene $\left(\right.$ GluRIIA $\left.^{\text {sp16}}\right)$ cause a significant decrease in the amplitude of spontaneous mEPSPs and a corresponding increase in presynaptic vesicle release (quantal content) (Petersen et al., 1997). In combination with the results observed in several other studies, it is concluded that a homeostatic increase in presynaptic release offsets the decrease in mEPSP amplitude caused by the mutation of the muscle-specific GluRIIA receptor subunit (for review, see Davis, 2006).

Here, we demonstrate that a heterozygous $g s b^{01155} /+$ mutation significantly suppresses the magnitude of synaptic homeostasis that is expressed in a GluRIIA ${ }^{s p 16}$ mutant background (Fig. 1A,B). We first demonstrate that synaptic transmission is normal in the heterozygous $g s b^{01155} /+$ mutant (Fig. 1). Specifically, we find that the wild-type average mEPSP amplitude (0.94 \pm 0.04 $\mathrm{mV} ; n=25)$ and average quantal content $(41.8 \pm 1.8 ; n=25)$ are not significantly different from the average mEPSP amplitude $(0.88 \pm 0.04 \mathrm{mV} ; n=23)$ and average quantal content $(45.3 \pm 1.9 ; n=23)$ recorded in $g s b^{01155} /+(p>0.1)$. However, when the heterozygous $g s b^{01155} /+$ mutation is placed in the background of the GluRIIA ${ }^{\text {sp16 }}$ mutant, we find that the homeostatic increase in presynaptic release that is normally observed in the GluRIIA $^{\text {sp16 }}$ animals is significantly suppressed (Fig. $1 B$ ). Specifically, the GluRIIA ${ }^{\text {sp } 16}$ mutant normally shows a $64 \%$ decrease in quantal size and a compensatory $98 \%$ increase in presynaptic release (quantal content). By contrast, when $g s b^{01155} /+$ is placed in the GluRIIA mutant, there is a $62 \%$ decrease in mEPSP amplitude but only a $37 \%$ increase in quantal content. Thus, the homeostatic enhancement of presynaptic release has been significantly suppressed $(p<0.003$ ) (Fig. 1). We have also quantified $g s b$ mRNA levels by quantitative (QT) PCR (see Materials and Methods) in $g s b^{01155} /+$ and find a significant $43 \pm 3 \%$ decrease in message $(p<0.01 ; n=3)$ compared with wild type, consistent with the haplo-insufficient phenotype observed in these animals. It is worth noting that it is not unusual for Paxfamily transcription factors to show haplo-insufficient mutant phenotypes (Wehr and Gruss, 1996; Dahl et al., 1997). In human, mutation of a single copy of pax3 is associated with Waardenburg syndrome (Waardenburg, 1951; Baldwin et al., 1992; Tassabehji et al., 1992, 1993; Dahl et al., 1997).

Gsb is widely expressed in the postembryonic nervous system To investigate how Gsb might participate in synaptic homeostasis, we examined the postembryonic expression of the $g s b$ gene. Previous reports have characterized $g s b$ expression during embryonic stages and have shown that this segment polarity gene specifies cell fate within a subpopulation of central neurons (Gutjahr et al., 1993; Li and Noll, 1993; Zhang et al., 1994). To visualize gsb expression in the larval CNS, we took advantage of the $g s b^{01155}$ transposon insertion. This transposon includes the $\beta$-galactosidase coding sequence and is inserted at position -46 relative to the transcription start site of the gsb gene (Duman-Scheel et al., 1997). Prior genetic studies demonstrate that this mutation specifically disrupts the $g s b$ gene, including analysis of $g s b^{01155}$ in 

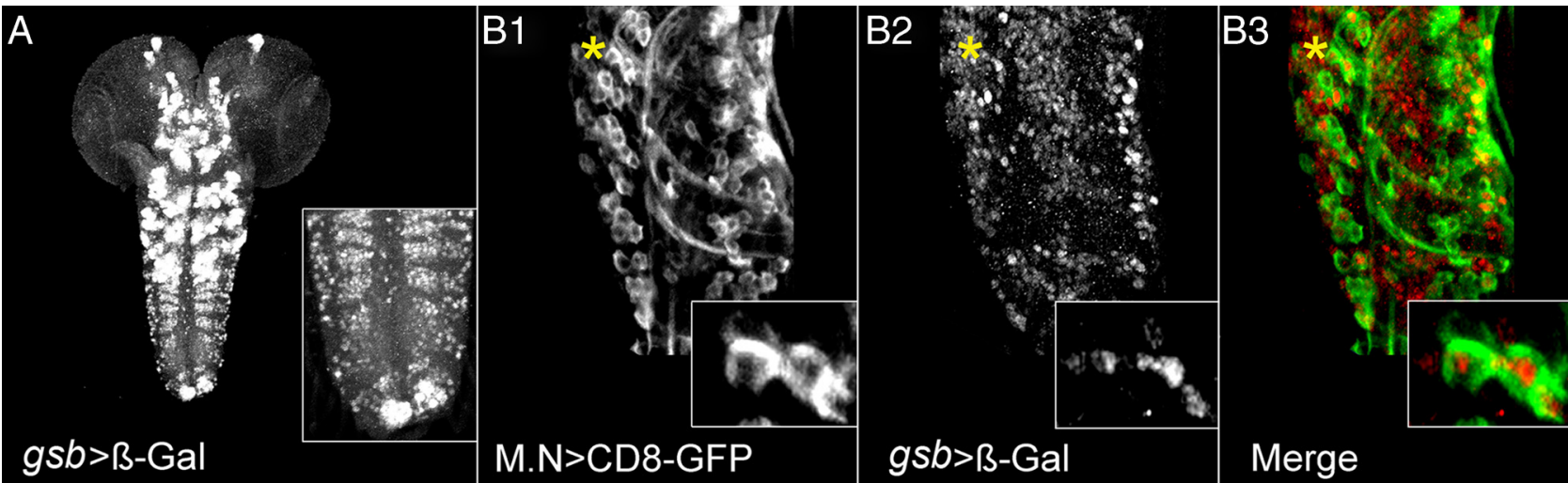

Figure 2. Gooseberry is expressed in mature motoneurons. $\boldsymbol{A}$, Whole mount CNS from a third instar larvae expressing $\beta$-galactosidase under the control of the $g$ s $b$ promoter shows that $g$ s $b$ is widely expressed throughout the CNS. The inset shows a region of the ventral nerve cord where motoneurons are located. This is also a prominent site of Gsb expression. $\boldsymbol{B}$, Whole mount CNS from a third instar larvae expressing $\beta$-galactosidase under the control of the gsb promoter (B2) and coexpressing CD8-GFP under a motoneuron (M.N)-specific driver (B1). These markers show colocalization in a subpopulation of motoneurons postembryonically. Asterisks indicate regions magnified for insets.

trans to a separate $g s b$ allele, reversion of the mutant phenotype following imprecise excision of the $g s b^{01155} \mathrm{P}$-element, and rescue of the mutant phenotype with a genomic fragment containing the $g s b$ region (Duman-Scheel et al., 1997). These and other data also support the conclusion that $\beta$-galactosidase reporter activity of this P-element insertion accurately reflects $g s b$ transcription (Duman-Scheel et al., 1997).

We have taken advantage of this P-lacZ insertion element $\left(g s b^{01155}\right.$ ) and document Gsb reporter expression throughout the CNS of the third instar larvae. It appears that $g s b$ is expressed in a large numbers of cells, more so than observed in the embryo, and is expressed in these cells throughout larval development. Importantly, we never observed $g s b$ expression in muscle by this method. We then combined the $g s b^{01155}$ reporter with a UASGFP (green fluorescent protein) reporter driven by a motoneuron-specific GAL4 driver. This allowed us to identify those motoneurons that express $g s b$. We find $\beta$-galactosidase immunoreactivity in the majority of motoneurons identified by UAS-GFP expression (Fig. 2) (98 $\pm 1.32 \%$ of motoneurons had $\beta$-galactosidase immunoreactivity levels higher than background levels, and $84 \%$ of these motoneurons showed immunofluorescence intensity $>2$ SDs above background). From these data we conclude that $g s b$ is expressed postembryonically in the majority of motoneurons, where it could participate in the mechanisms of synaptic homeostasis.

\section{Gsb is required postembryonically for the expression of} synaptic homeostasis

We hypothesize that postembryonic $g s b$ expression is required for the expression of synaptic homeostasis. To test this hypothesis, we engineered animals that express a gene-specific $g s b$-RNAi under the control of the UAS promoter (UAS-gsb-RNAi). First, we performed experiments to test whether UAS-gsb-RNAi could inhibit $g s b$ expression. We initially performed a functional assay of Gsb activity. When gsb is overexpressed in the developing wing, there is a severe perturbation of wing morphology $(100 \%$ penetrance), consistent with the function of $g s b$ as a potent regulator of developmental signaling (Fig. 3). However, when we coexpress UAS-gsb with UAS-gsb-RNAi, we are able to significantly suppress this morphological defect, resulting in nearly normal wing morphology with $100 \%$ penetrance. These data are consistent with $U A S-g s b-R N A i$ limiting $g s b$ expression. We then quantified $g s b$ mRNA levels in animals neuronally expressing

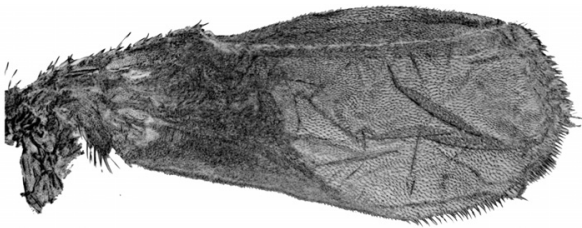

MS1096-Gal4/+; +; UAS-gsb/+

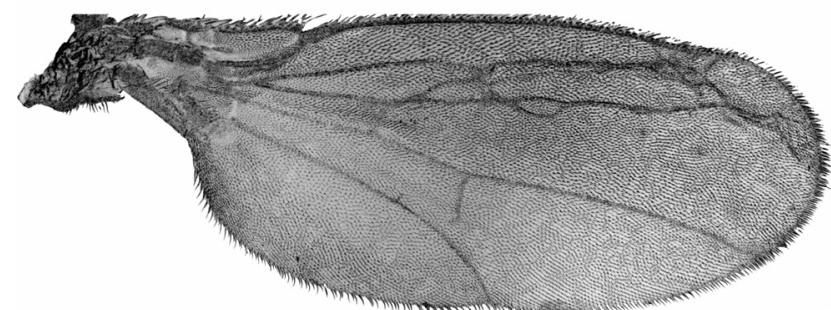

MS1096-Gal4/+; UAS-gsb-RNAil+; UAS-gsb/+

Figure 3. Transgenically expressed UAS-gsb-RNAi can reduce gsb activity in vivo. Top, Overexpression of UAS-Gsb is driven by the wing-pouch driver MS-1096 GAL4 line and provokes profound wing anomalies with $100 \%$ penetrance. Bottom, Coexpression of UAS-gsb-RNAi restores wings morphology toward a wild-type phenotype ( $100 \%$ penetrance) demonstrating that expression of UAS-gsb-RNAi effectively reduces gsb activity.

$U A S-g s b-R N A i$ by QT-PCR and find a significant $48 \pm 2 \%$ decrease in $g s b$ mRNA compared with controls $(n=3 ; p<0.01)$.

Next, we assayed synaptic function when UAS-gsb-RNAi is expressed in postmitotic neurons using the pan-neuronal GAL4 driver elav-GAL4. There is no statistically significant difference when comparing recordings made in wild type to those made in animals with neuronal expression of UAS-gsb-RNAi (elav-Gal4; $U A S-g s b-R N A i)$. The average wild-type mEPSP amplitude is $0.96 \pm 0.04 \mathrm{mV}(n=19)$ (Fig. $4 A)$, and quantal content is $35.5 \pm$ $1.6(n=19)$ (Fig. 4A). When UAS-gsb-RNAi is expressed in postmitotic neurons we find that mEPSPs are $0.94 \pm 0.05 \mathrm{mV}$ $(n=9)$ and quantal content is $33.4 \pm 1.9(n=23)$ (Fig. $4 B)$. Thus, RNAi-dependent knockdown of $g s b$ does not alter baseline synaptic transmission.

We then asked whether neuronal expression $U A S-g s b-R N A i$ disrupts synaptic homeostasis in the GluRIIA mutant background. We find that expression of UAS-gsb-RNAi in the 
A

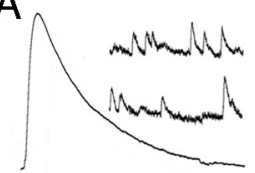

wild type

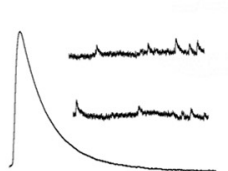

GIURIIA

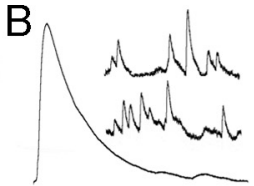

elav-Gal4

UAS-gsb-RNAi

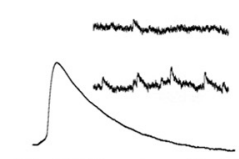

GluRIIA; elav-Gal4 UAS-gsb-RNAi

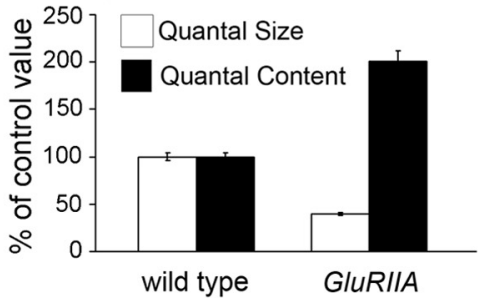

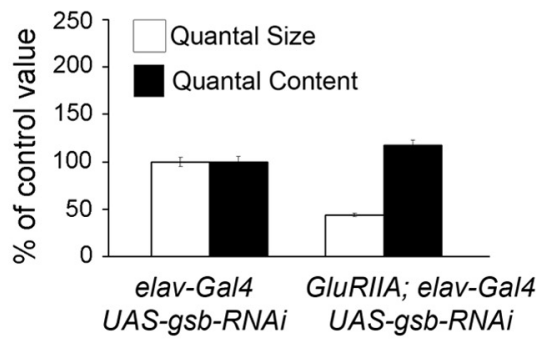

C

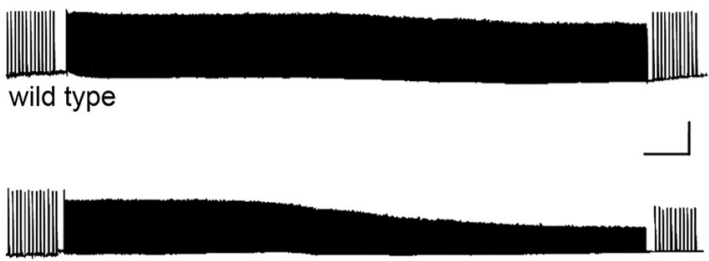

GIURIIA

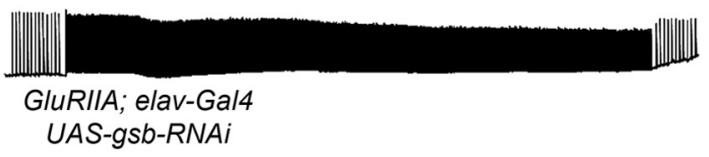

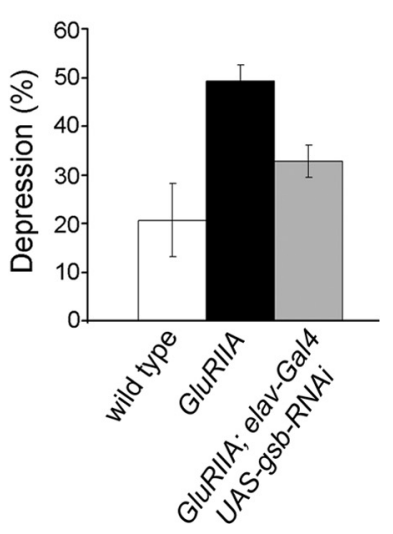

Figure 4. Disrupting gsb expression in postmitotic neurons strongly diminishes synaptic homeostasis. $\boldsymbol{A}$, Representative traces of wild type and GluRIII $A^{\text {sp16 }}$ and quantification of average mEPSP amplitude (open bars) and quantal content (filled bars). Values are normalized to wild-type control. GluRIII $A^{\text {sp16 }}$ mutants have decreased mEPSP amplitudes and a homeostatic increase in quantal content $(p<0.001)$. B, Representative elav-Gal4; UAS-gsb-RNAi and GluRIIA; elav-Gal4; UAS-gsb-RNAi. Note that there is no statistically significant difference between wild-type mEPSPs $(0.96 \pm 0.04 \mathrm{mV} ; n=19 ;$ in $A)$ and quantal content $(35.5 \pm$ $1.6 ; n=19 ;$ in $A)$ and elav-Gal4; UAS-gsb-RNAi mEPSPs $(0.94 \pm 0.05 \mathrm{mV} ; n=9)$ and quantal content $(33.4 \pm 1.9 ; n=9 ; p=$ 0.43). Quantal content (filled bar) and mEPSP amplitude (open bar) are quantified. GluRIIA; elav-Gal4; UAS-gsb-RNAi animals are unable to express a homeostatic increase in quantal content despite a significant decrease in mEPSP amplitude. GluRIIIA ${ }^{5 p 16}$ mutants animals (mEPSP $=0.38 \pm 0.02 \mathrm{mV} ; \mathrm{EPSP}=26.5 \pm 1.4 \mathrm{mV} ; \mathrm{QC}=71.2 \pm 3.9 ; n=19)$ showed robust homeostatic compensation (QC is increased by $101 \pm 11 \%$ compared with control value; $p=4.710^{-9}$ ), while elav-Gal4; GluRIIIAp16; UASgsb-RNAi/ + animals ( $\mathrm{mEPSP}=0.41 \pm 0.02 \mathrm{mV} ; \mathrm{EPSP}=16.1 \pm 0.9 \mathrm{mV} ; \mathrm{QC}=39.2 \pm 1.9 ; n=9$ ) showed a near complete blockade of synaptic homeostasis ( $Q$ C is increased by $17 \pm 6 \%$ compared with control value; $p=5.810^{-8}$ when compared with GluRIIA compensation, $p=0.048$ when compared with elav-Gal4; UAS-gsb-RNAi). C, Sample traces showing that there is significantly greater depression of EPSP amplitudes in GluRIIA mutant synapses during sustained stimulation in elevated extracelIular calcium ( 6000 stimuli at $8 \mathrm{~Hz}$ in $2 \mathrm{~mm} \mathrm{Ca}{ }^{2+}$ ). This effect is strongly reduced in GluRIIA; elav-Gal4; UAS-gsb-RNAi synapses, consistent with a decreased release probability in these animals. Rapid recovery to initial EPSP amplitudes is demonstrated by low-frequency stimulation $(0.2 \mathrm{~Hz})$ following the cessation of the stimulus train. Right, Quantification of the depression at the end of the train shows that removing gsb activity in GluRIIA mutant animals significantly reduces synaptic depression. GluRIIIA animals show a $49.3 \pm 3.4 \%$ depression while control animals show $20.7 \pm 7.5 \%$ depression and GluRIIA; elav-Gal4; UAS-gsb-RNAi animals show a $32.8 \pm 3.3 \%$ depression. Scale bar, $1 \mathrm{~min} / 25 \mathrm{mV}$. Experiments in C were performed in high extracellular calcium $(2 \mathrm{mM})$. At this concentration, the amplitude of the EPSP does not directly reflect quantal content due to the effects of nonlinear summation, and there is no difference in initial EPSP amplitude comparing GluRIIA and GluRIIA/RNAi. Error bars represent average \pm SEM. formed a control examining musclespecific expression of UAS-gsb-RNAi and find that synaptic homeostasis is unchanged compared with wild type (GluRIIA; BG57-Gal4; UAS-gsb-RNAi compared with wild type; $n=15 ; p=$ $0.67)$. These data confirm that loss of Gsb disrupts the expression of synaptic homeostasis. In addition, these data demonstrate that $g s b$ is necessary within postmitotic neurons for the expression of synaptic homeostasis. This represents a novel postembryonic function for this well documented developmental patterning gene.

Synaptic homeostasis at the Drosophila NMJ is expressed as an increase in presynaptic transmitter release. To further verify that expression of $U A S-g s b-R N A i$ disrupts a homeostatic increase in presynaptic release probability, we assayed shortterm synaptic plasticity at the NMJ. The wild-type NMJ is able to maintain EPSP amplitudes without substantial synaptic depression during prolonged synaptic stimulation in elevated extracellular calcium saline ( $2 \mathrm{mM} \mathrm{Ca}^{2+}$ ) (Marie et al., 2004; Frank et al., 2006). By contrast, GluRIIA mutant animals show pronounced synaptic depression under identical conditions, most likely because the increased rate of vesicle release in the GluRIIA mutant exceeds the rate of vesicle endocytosis, resulting in vesicle depletion (Frank et al., 2006). If loss of $g s b$ prevents a homeostatic increase in presynaptic release in the GluRIIA mutant background, then it should also relieve the synaptic depression normally observed in the GluRIIA mutant background. This is confirmed here. We demonstrate that expression of UAS-gsbRNAi in the background of the GluRIIA mutant significantly reduces the synaptic depression normally observed in the GluRIIA mutant (Fig. 4C). Synaptic depression is not restored completely to wild-type levels. However, on average there remains a small amount of homeostatic compensation in GluRIIA animals that also express $U A S-g s b-R N A i$. Together, these data are consistent with the conclusion that neuronal expression of Gsb-RNAi disrupts the homeostatic increase in presynaptic release normally observed in the GluRIIA mutant background.
GluRIIA mutant background (elav-Gal4; GluRIIA ${ }^{\text {sp16; }}$ UAS-gsb$R N A i /+$ ) significantly suppresses the homeostatic increase in presynaptic release normally observed in the GluRIIA mutant (a homeostatic increase in quantal content of only $17 \%$ was found in animals expressing UAS-gsb-RNAi compared with a $101 \%$ increase observed in the GluRIIA mutant alone) (Fig. $4 B)$. Although $g s b$ is not expressed in muscle, we also per-
Loss of postmitotic Gsb does not alter NMJ growth and morphology

It is possible that loss of gsb could lead to abnormal synaptic growth and/or development and indirectly impair synaptic homeostasis. Therefore, we assessed NMJ morphology and quantified the growth of the NMJ by counting the number of synaptic boutons present at an identified NMJ. There is no change in the 

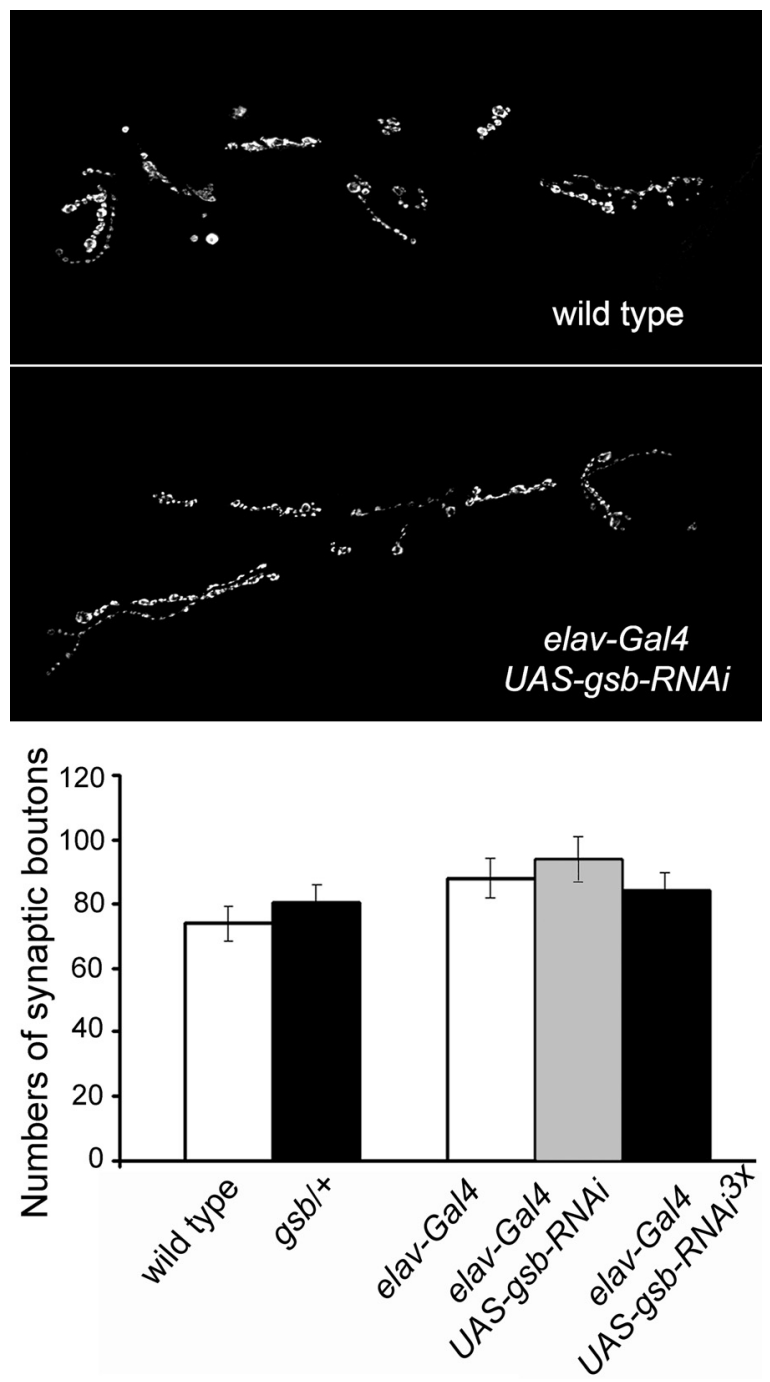

Figure 5. NMJ growth is normal following neuronal gsb knockdown. Top, Images of NMJs at muscles 6/7 of wild type and UAS-gsb-RNAi expressed presynaptically (elav-Gal4; UAS-gsbRNAi). Bottom, Quantification of NMJ size at muscles 6 and 7 within segment $A 3$ of third instar larvae. No significant difference in bouton number was observed comparing wild type with $g s b /+$ or when comparing animals expressing UAS-gsb-RNAi with the elav-Gal4 controls ( $n>$ 8). Error bars represent mean \pm SEM.

morphology of the NMJ in animals with decreased levels of $g s b$. In particular, there is no evidence of altered bouton size or "satellite" bouton formation (Marie et al., 2004). In addition, there is no significant difference between control NMJs and NMJs with decreased $g s b$, either in the heterozygous mutant background or in animals expressing UAS-gsb-RNAi in postmitotic neurons (Fig. 5).

Next, we asked whether essential synaptic antigens are correctly organized and expressed in motoneurons with decreased $g s b$ activity. To do so, we examined several different synaptic markers and asked whether the distribution or abundance of these markers was altered in neurons with decreased $g s b$. We examined Bruchpilot, a protein homologous to ELKS/CAST that promotes active zone assembly and vesicle release (Kittel et al., 2006; Wagh et al., 2006), Dynamin (Roos and Kelly, 1998; Sever, 2002), Synapsin (Klagges et al., 1996; Fdez and Hilfiker, 2006), and Dlg (Budnik et al., 1996; Hough et al., 1997). Decreased gsb levels do not alter the subcellular localization or the abundance of any of these proteins at the NMJ
(Fig. 6). Thus, we conclude that loss of $g s b$ in postmitotic neurons does not have a significant effect on the development or growth of the NMJ.

\section{Loss of $g s b$ does not impair the rapid induction of synaptic homeostasis}

Application of a sub-blocking concentration of the glutamate receptor antagonist $\mathrm{PhTx}$ causes a rapid decrease in mEPSP amplitude and a corresponding homeostatic increase in presynaptic transmitter release (Frank et al., 2006). The homeostatic increase in release induced by acute application of PhTx occurs rapidly, being observed in 2-10 $\mathrm{min}$, demonstrating a rapid induction of synaptic homeostasis at the Drosophila NMJ (Frank et al., 2006). Several experiments suggest that the mechanisms responsible for the rapid induction of synaptic homeostasis by $\mathrm{PhTx}$ are similar to the mechanisms that are responsible for a homeostatic increase in presynaptic release in the GluRIIA mutant animal. For example, mutations in the cacophony calcium channel $\left(\mathrm{Ca}_{\mathrm{V}} 2.1\right.$ homolog) prevent a homeostatic increase in presynaptic release in both the GluRIIA mutant background and following PhTx application to the NMJ (Frank et al., 2006). Similarly, mutations in the BMP signaling system disrupt synaptic homeostasis in both the GluRIIA mutant and following PhTx application to the NMJ (Goold and Davis, 2007). Therefore, we asked whether loss of $g s b$ also inhibits the rapid PhTx-dependent induction of synaptic homeostasis.

We applied PhTx (4 $\mu \mathrm{M})$ to the Drosophila NMJ as done previously (Frank et al., 2006; Goold and Davis, 2007). In wild type, $g s b^{01155} /+$ mutants, and animals expressing UAS-gsb-RNAi in neurons, the application of PhTx causes an indistinguishable decrease in average mEPSP amplitude and a homeostatic increase in presynaptic release (Fig. 7). Thus, the rapid induction of synaptic homeostasis is unaffected in the same $g s b$ loss-of-function conditions that are sufficient to inhibit synaptic homeostasis in the GluRIIA mutant background. Next, we sought to knock down gsb expression as much as possible. We have previously found that expression of multiple copies of a UAS-RNAi transgene in neurons is necessary to achieve near complete depletion of protein from motoneurons at the Drosophila NMJ (Pielage et al., 2005, 2008). Therefore, we applied PhTx to flies containing the postmitotic driver elav ${ }^{\mathrm{Cl} 55}$-Gal4, directing the expression of two or three inserts encoding for UAS-gsb-RNAi (Fig. 7). After PhTx application, the mEPSP amplitudes are similarly decreased in control animals and in elav ${ }^{C 155}$-Gal4; UAS-gsb-RNAi ${ }^{2 \times}$ or

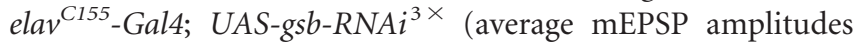
are, respectively, 56.6, 57.1, and 53\% of the control amplitude) (Fig. 7). Again, all three genotypes also showed robust homeostatic compensation (an increase of 67, 57, and 83\% above the control QC) (Fig. 7) that is not significantly different from that observed following PhTx application to the genetic controls (Fig. 7). From these data we conclude that $g s b$ is not required for the rapid induction of synaptic homeostasis. Ultimately, we cannot rule out the possibility that synaptic homeostasis in the GluRIIA mutant background is simply more sensitive to decreased levels of Gsb protein compared with the mechanisms underlying the rapid $\mathrm{PhTx}$-dependent induction of synaptic homeostasis. However, we favor the conclusion that $g s b$ selectively participates in a mechanism that is involved in the sustained expression of synaptic homeostasis versus the rapid protein synthesis-independent induction of synaptic homeostasis. 

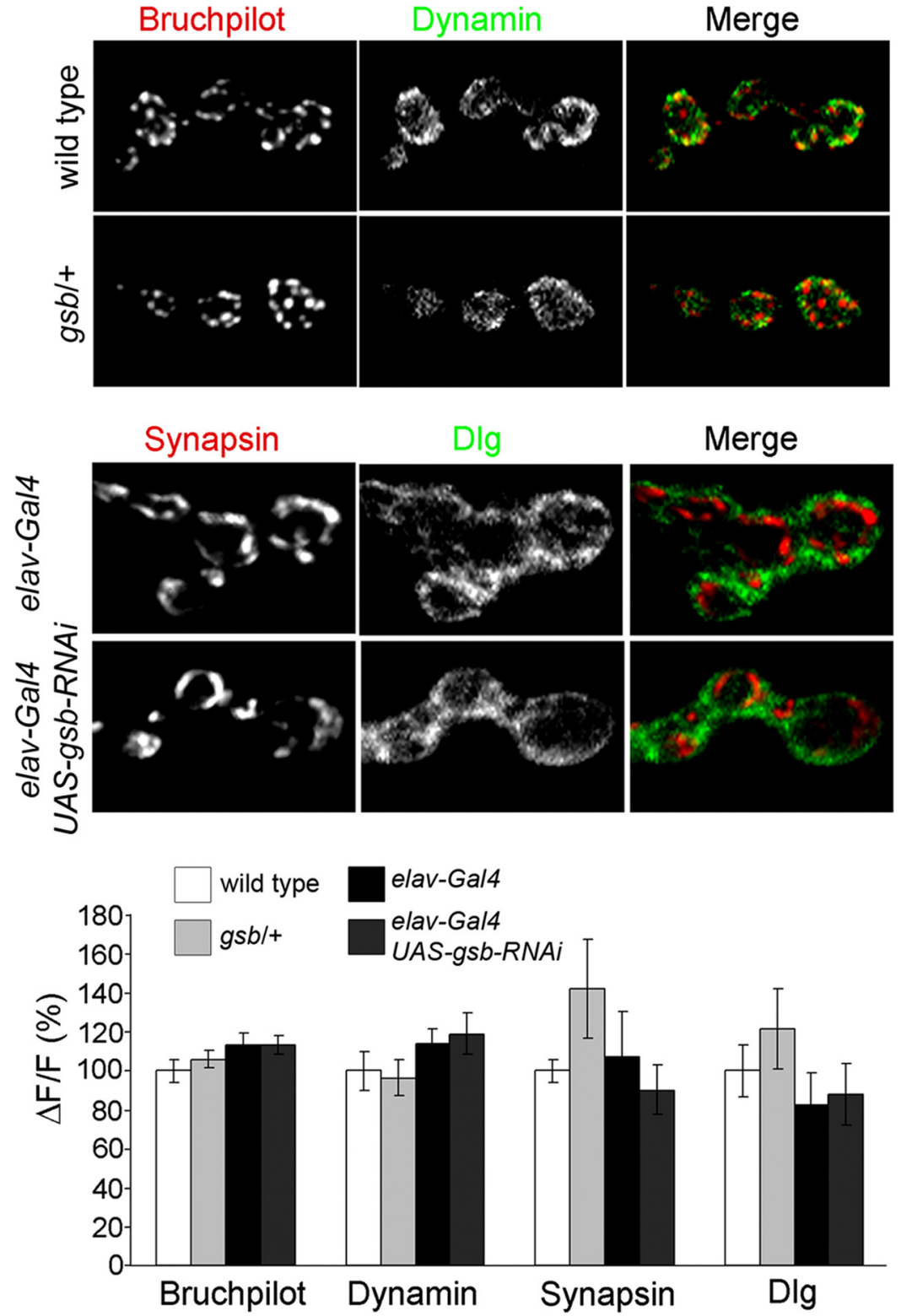

Figure 6. Decreased gsb does not affect the abundance or localization of synaptic antigens. Top, Representative wild-type and gsb $b^{01155}$ / + synaptic boutons at muscle 4 (segment A3) show that Bruchpilot (red) and Dynamin (green) localization and abun-

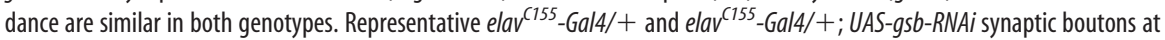
muscle 4 (segment A3) show that Synapsin (red) and Dlg (green) localization and abundance are similar in both genotypes. Bottom, Quantification of the fluorescence intensity for Bruchpilot, Dynamin, Synapsin, and Dlg at the NMJ of muscle 4 ( $n>7$ for each genotype). The abundance of these markers is not statistically different in any of the genotypes tested. Values are normalized to wild-type control. Error bars represent average \pm SEM.

\section{Gsb is not required for homeostatic signaling that decreases} presynaptic release

Two recent studies have shown that homeostatic signaling at the Drosophila NMJ is also capable of decreasing presynaptic release in response to perturbations that either increase muscle innervation (Davis and Goodman, 1998) or increase presynaptic quantal size (Daniels et al., 2004). For example, neuronal expression of the vesicular glutamate transporter (vGlut) causes an increase in quantal size that is attributed to an increase in the amount of neurotransmitter packaged into individual synaptic vesicles at the Drosophila NMJ (Daniels et al., 2004). In response to increased quantal size, there is a compensatory decrease in quantal content that restores average EPSP amplitudes to wild-type levels.
It remains unknown whether a homeostatic decrease in presynaptic release requires the same molecular machinery as the homeostatic increase in presynaptic release observed in the GluRIIA ${ }^{\text {sp16 }}$ mutant background (Davis, 2006). Therefore, we generated animals that coexpress the vGlut transporter and UAS-gsb-RNAi in postmitotic neurons. We find that expression of the vGlut transporter in neurons caused a significant increase in average mEPSP amplitude when driven alone $(54 \%$ increase beyond control mEPSP amplitude) or in combination with the UAS- $g s b-R N A i$ (47\% increase beyond control mEPSP amplitude) (Fig. 8). We also find that there is a homeostatic decrease in presynaptic quantal content when UAS- $v$ Glut is driven alone $(77.2 \%$ of control QC) or in combination with UAS-gsb-RNAi (78.7\% of control QC) (Fig. 8). These data indicate that $g s b$ is not required for a homeostatic decrease in presynaptic release.

One possibility is that coexpression of two UAS constructs dilutes the transgene expression, making the UAS-gsb-RNAi less effective. However, the efficacy of the $U A S-v G l u T$ transgene in generating a change in quantal size is identical regardless of whether UAS-gsb-RNAi is coexpressed ( $\mathrm{mEPSP}$ amplitudes are increased identically). This suggests that there is no quantitative "dilution" effect from coexpressing two UAS constructs. Based upon these data, we conclude that Gsb is necessary only for the homeostatic increase in release that occurs following a decrease in mEPSP amplitude and is dispensable for homeostatic mechanisms that decrease presynaptic release in response to increased mEPSP amplitude.

As a final control, we considered the possibility that postembryonic expression of $g s b$ is necessary to maintain the general electrophysiological profile of Drosophila motoneurons. Since $g s b$ is a transcription factor involved in neuronal cell fate specification, we reasoned that postembryonic Gsb might be important for maintaining motoneuron firing properties and that this could influence the expression of synaptic homeostasis. For example, a chronic decrease in motoneuron excitability might initiate a homeostatic enhancement that could occlude further homeostatic regulation in the GluRIIA mutant background. Therefore, we recorded from motoneuron soma in the CNS following a series of step depolarizations of increasing amplitude (Fig. 9A). Step depolarizations of $10-50 \mathrm{pA}$ elicited action potential trains that did not differ comparing wild type and animals expressing UAS- $g s b$ $R N A i(p>0.1)$. Depolarizations between 60 and $100 \mathrm{pA}$ elicited action potential trains of slightly, although statistically significant, higher frequency in motoneurons expressing UAS-gsb$R N A i$ compared with controls. However, there was no significant 


$$
\text { elav-Gal4; UAS-gsb-RNA/3x }
$$

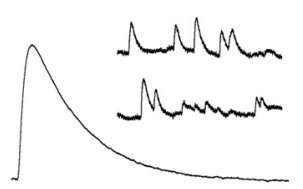

- PTOX
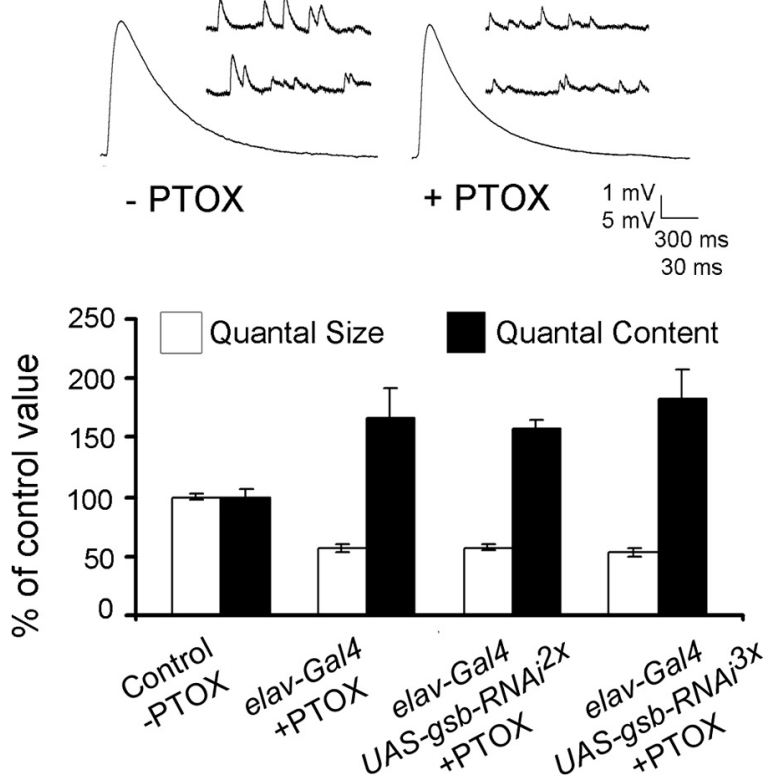

Figure 7. Evidence that $g s b$ is not required for the rapid induction of synaptic homeostasis. Top, Representative traces for elav-Gal4; UAS-gsb-RNAi ${ }^{\times}$animals with and without PhTx (PTOX). Bottom, Quantal content (filled bar) and mEPSP amplitude (open bar) are quantified. The data represent values recorded after $10 \mathrm{~min}$ of $\mathrm{PhTx}$ application and are presented normalized to control values recorded in the absence of PhTx. There is a significant decrease in $\mathrm{mEPSP}$ amplitude and a significant compensatory increase in quantal content for the three genotypes. Error bars represent mean \pm SEM.

change in the individual action potential waveform (Fig. $9 B-E$ ). Since the observed difference in UAS-gsb-RNAi-expressing animals is small, occurs only at the largest step depolarizations, causes an increase rather than a decrease in firing rate, and occurs without a change in action potential waveform, we favor the conclusion that impaired Gsb-dependent maintenance of motoneuron firing properties is unlikely to be the direct cause of impaired synaptic homeostasis.

\section{Evidence that Gsb antagonizes Wingless signaling to allow} synaptic homeostasis

Finally, we sought to provide insight into the mechanism by which Gsb might influence the sustained expression or consolidation of synaptic homeostasis. To gain insight into the signaling pathways influenced by Gsb, we turned to studies of embryonic Gsb function. During embryonic patterning, $g s b$ functions cell autonomously to suppress the action of Wingless $(\mathrm{Wg})$. In brief, $g s b$ acts specifically within the $\mathrm{Wg}$ secreting cell to suppress autocrine Wg signaling, allowing Wg-dependent intercellular regulation of cell fate (Duman-Scheel et al., 1997; Bhat et al., 2000) (Fig. $10 \mathrm{~A}$ ). In the vertebrate and invertebrate nervous systems there is evidence that $\mathrm{Wg}$ is secreted by neurons and functions as a potent intercellular signaling molecule (Packard et al., 2002; Charron and Tessier-Lavigne, 2005; Davis et al., 2008; Henriquez et al., 2008; Miech et al., 2008; Poon et al., 2008; Gogolla et al., 2009; Jing et al., 2009; Korkut and Budnik, 2009). At the Drosophila NMJ, Wg is secreted by the motoneuron and has been shown to act postsynaptically via the DFrizzled2 receptor (Packard et al., 2002; Mathew et al., 2005). Wg has also been shown to act upon the presynaptic nerve terminal to sculpt the morphology of the nerve terminal (Miech et al., 2008). Presynaptic effects of Wg are
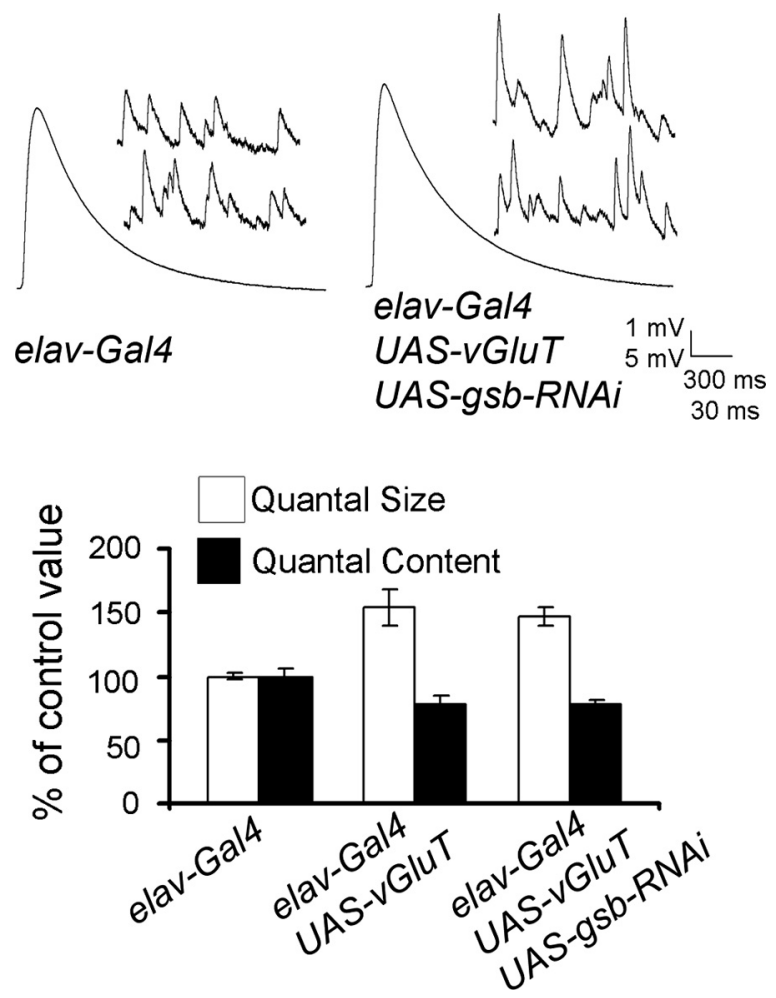

Figure 8. Evidence that $g s b$ is not required for the homeostatic response to increased quantal size. Top, Representative traces for elav-Gal4 and elav-Gal4; UAS-vGluT/+; UAS-gsb-RNAi/ + . Bottom, Quantal content (filled bar) and mEPSP amplitude (open bar) are quantified and normalized to control values (elav-Gal4). There is a significant increase in quantal size and a significant, compensatory decrease in quantal content in elav-Gal4; UAS-VGluT/+ and in elavGal4; UAS-vGluT/+; UAS-gsb-RNAi/+ animals. Error bars represent mean \pm SEM.

mediated by the Arrow receptor and includes signaling that is localized to the presynaptic nerve terminal (Miech et al., 2008).

We first hypothesized that the embryonic function of Gsb might be conserved in postembryonic motoneurons and, therefore, that Gsb might act to inhibit Wingless signaling in motoneurons. Next, we considered that loss of Gsb suppresses synaptic homeostasis. Therefore, if Gsb inhibits Wg signaling, then Wg would likely function as an endogenous inhibitor of homeostatic compensation. Thus, the persistent activity of Gsb would be necessary to block the action of $\mathrm{Wg}$ and enable the expression of synaptic homeostasis. This model predicts, therefore, that loss of $w g$ should restore homeostatic compensation to the GluRIIA; $g s b /+$ double mutant animals (Fig. 10A).

We generated double and triple mutant animals in which we combined a $w g$ mutation with the GluRIIA and $g s b$ mutations. In these experiments, we took advantage of a well characterized, loss-of-function $w g$ mutation, $w g^{I-12}$ (C104S) (van den Heuvel et al., 1993, Couso and Martinez Arias, 1994), shown previously to alter NMJ development in Drosophila (Packard et al., 2002). In our experiments we sought to decrease but not eliminate $\mathrm{Wg}$ signaling. Therefore, we analyzed heterozygous $\mathrm{wg}^{\mathrm{I}-12}$ mutants. NMJ development is normal in these animals, allowing us to circumvent a requirement of Wg during anatomical NMJ development. We first show that synaptic homeostasis occurs normally in GluRIIA animals harboring a heterozygous $w g^{I-12}$ mutation (Fig. 10B). Indeed, there appears to be no effect on synaptic homeostasis or baseline synaptic release properties in these animals. Next, we examined the triple mutant combination that includes a heterozygous $w g^{I-12}$ mutation, the homozygous 
A
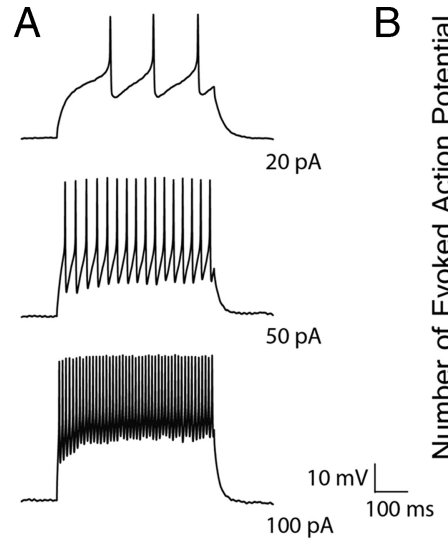

B

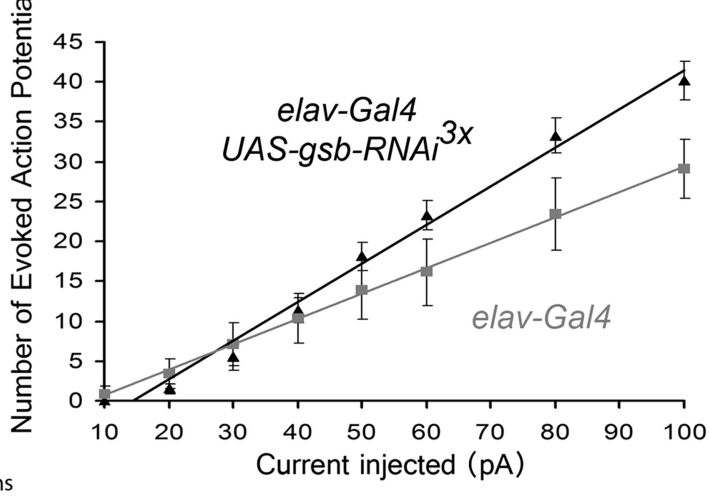

C

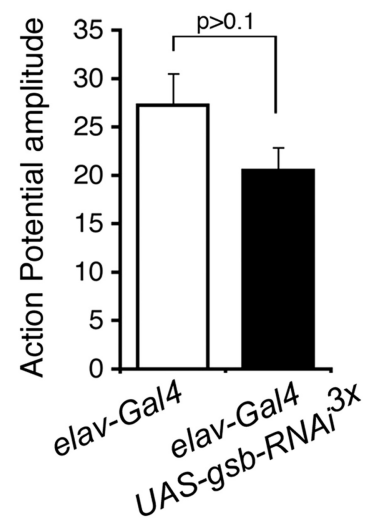

D

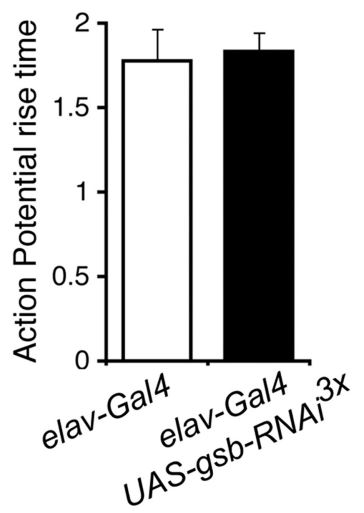

E

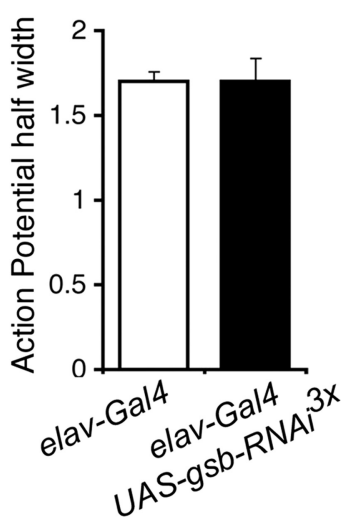

Figure 9. Motoneuron excitability in animals expressing UAS-gsb-RNAi. $\boldsymbol{A}$, Representative traces recorded from motoneuron soma in response to the indicated step depolarization. $\boldsymbol{B}$, Quantification of action potential number across the range of step depolarizations tested (current injected) for control (elav-Gal4) and animals expressing UAS-gsb-RNAi (elav-Gal4; UAS-gsb$\left.R N A i^{\times}{ }^{\times}\right) . \boldsymbol{C}-\boldsymbol{E}$, There is no significant difference in action potential amplitude $(\boldsymbol{C})$, rise time $(\boldsymbol{D})$, or half-width $(\boldsymbol{E})$ comparing control (elav-Gal4) and animals expressing elav-Gal4; UAS-gsb-RNAi ${ }^{\times}$.

GluRIIA mutation, and a heterozygous gsb mutation. There is significantly more homeostatic compensation in the triple mutant compared with the GluRIIA; gsb/+ double mutants. Indeed, the presence of the heterozygous $w g^{I-12} /+$ mutation restores homeostatic compensation to levels that are statistically the same as those observed in GluRIIA alone (Fig. 10C). These data are consistent with a model in which Gsb has a conserved function to suppress Wg signaling in Drosophila motoneurons. Furthermore, these data suggest the interesting possibility that an additional function for Wg signaling at the Drosophila NMJ is to inhibit homeostatic compensation.

\section{Discussion}

In this study we advance our understanding of synaptic homeostasis in several important ways. First, we identify a new gene, $g s b$, that is required in postmitotic, postembryonic neurons for synaptic homeostasis at the Drosophila NMJ. Drosophila gsb is the homolog of vertebrate pax3/pax7. Thus, our data identify a new function for a conserved gene family that has been traditionally studied in the context of neuronal fate specification. Second, we demonstrate that loss of $g s b$ selectively disrupts the expression of synaptic homeostasis without impairing the rapid induction of synaptic homeostasis. These data suggest that genetically separable mechanisms exist for the induction versus the expression of synaptic homeostasis at the Drosophila NMJ, consistent with recently published data (Frank et al., 2009).
Third, we demonstrate that loss of Gsb selectively disrupts the mechanisms responsible for a homeostatic increase in presynaptic release without impairing the mechanisms responsible for homeostatic decrease in presynaptic release. Therefore, these two forms of homeostatic modulation, both expressed at the Drosophila NMJ, appear to involve genetically separable mechanisms. Fourth, because $g s b$ is a transcription factor, our data highlight the possibility that the persistent expression of synaptic homeostasis in the GluRIIA mutant is consolidated through transcription-/translation-dependent mechanisms, while the rapid induction of homeostatic signaling is independent of new protein synthesis (Frank et al., 2006). Thus, there may exist genetically separable phases of homeostatic signaling at the Drosophila NMJ analogous to the induction versus expression of longterm synaptic plasticity in other systems. Finally, we present evidence to support the hypothesis that Gsb functions similarly during cell fate specification and synaptic homeostasis. According to the emerging model, Gsb may antagonize Wingless signaling in motoneurons to facilitate the consolidation of synaptic homeostasis. While there remains considerable work to prove this model, our data, in combination with prior work during embryonic cell fate specification, provide the basis for a compelling model that can be examined in greater detail in future studies.

Gsb selectively impairs the expression of synaptic homeostasis Here, we demonstrate that motoneurons with decreased levels of Gsb are unable to express synaptic homeostasis in the background of a GluRIIA mutation. By contrast, the rapid, protein synthesis-independent induction of synaptic homeostasis following application of the glutamate receptor antagonist PhTx is normal. One possible explanation for this difference is that $\mathrm{PhTx}$ and the GluRIIA mutant cause different postsynaptic perturbations and initiate separate homeostatic signaling systems, only one of which is affected by loss of $g s b$. This seems unlikely, however, because previously published data indicate that PhTx primarily acts upon postsynaptic glutamate receptors including those that contain the GluRIIA receptor subunit (Frank et al., 2006). Furthermore, several mutations have been shown to block synaptic homeostasis both in the GluRIIA mutant and following PhTx application, demonstrating that these two perturbations share, at some level, a common molecular mechanism of homeostatic signaling (Frank et al., 2006; Goold and Davis, 2007). We hypothesize, therefore, that loss of Gsb impairs a molecular process that is selectively involved in the sustained expression of synaptic homeostasis. This would be consistent with the sustained expression of synaptic homeostasis requiring new protein synthesis.

The possibility that Gsb participates specifically in the sustained expression or consolidation of synaptic homeostasis has interesting implications. In one model of homeostatic signaling, 
A

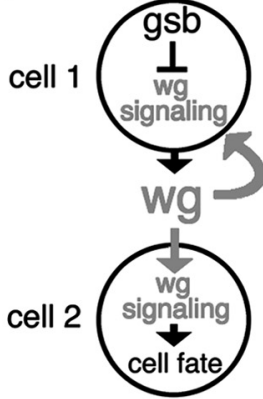

B

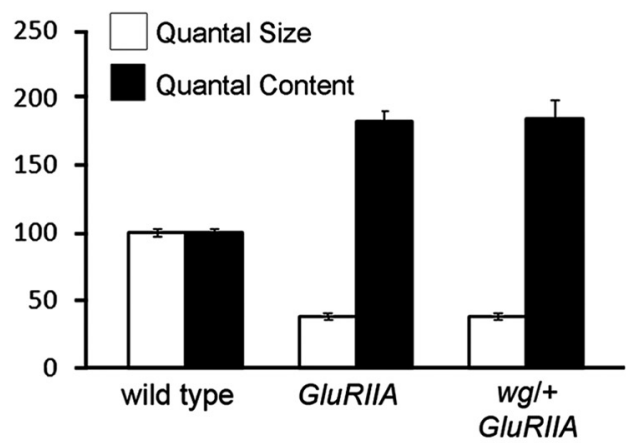

C

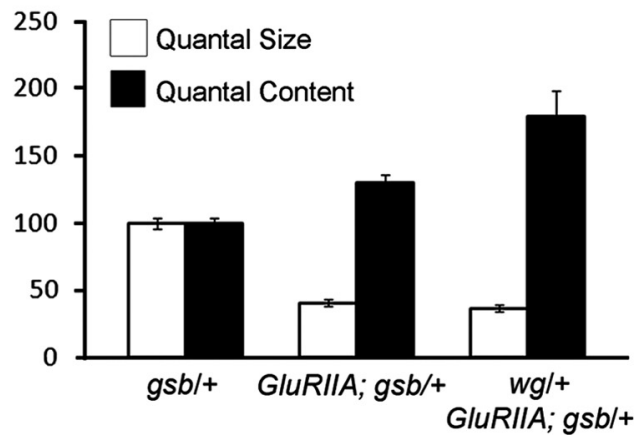

Figure 10. Synaptic homeostasis requires Gsb to antagonize Wg signaling. $A$, Left, During neuronal development, neuroblasts expressing Gsb are impervious to $\mathrm{Wg}$ autocrine signaling. Right, We envision that this signaling is also controlling the expression of synaptic homeostasis. $B$, Quantal content (filled bars) and mEPSP amplitude (open bars) are quantified and normalized to control values $\left(\mathrm{W}^{-}\right)$. GluRIII $A^{\mathrm{sp} 16}$ animals ( $\mathrm{mEPSP}=0.36 \pm 0.01 \mathrm{mV} ; \mathrm{EPSP}=26.2 \pm$ $1.1 \mathrm{mV}$; quantal content $=75 \pm 3.1 ; n=33$ ) show a considerable homeostatic increase in $Q C$ (an increase of $83 \% \pm 7.6 \%$ beyond control values; $p=7.610^{-13}$ ) when compared with wild-type values ( $\mathrm{mEPSP}=0.95 \pm 0.03 \mathrm{mV}$; EPSP $=37.9 \pm 0.67 \mathrm{mV}$; quantal content $=$ $41 \pm 1.3 ; n=35)$. GluRIIIS ${ }^{\text {sp16 }}$ mutants missing one copy of $w g\left(G / u R I I A^{5 p 16} ; w g^{1-12} /+\right)$ show a similar decrease in mEPSP amplitudes $(0.36 \pm 0.02 \mathrm{mV})$ and a compensatory homeostatic increase in quantal content $(E P S P=26.9 \pm 2.3 \mathrm{mV} ; Q \mathrm{QC}=75.5 \pm 5.9 ; \mathrm{QC}$ is increased by $84 \pm$ $14.4 \%$ compared with control values; $\left.p=10^{-4}\right)$. C, The GluRll/ ${ }^{\text {sp16 }}, g s b^{01155} /$ GluRIIIA ${ }^{\text {sp16 }}$, + animals show greatly reduced synaptic transmission (mEPSP $=0.35 \pm 0.01 \mathrm{mV}$; EPSP $=$ $20.5 \pm 1 \mathrm{mV} ; \mathrm{QC}=58.8 \pm 2.8 ; n=28$ ) when compared with $g \mathrm{sb}^{01155} /+$ control animals $(\mathrm{mEPSPs}=0.88 \pm 0.04 \mathrm{mV} ; \mathrm{EPSPs}=39.1 \pm 1.3 \mathrm{mV} ; \mathrm{QC}=45.3 \pm 1.9 ; n=23$ ). This is caused by compromised homeostasis compensation: $Q C$ is increased by only $30 \pm 6.2 \%$ compared with control values; $p=10^{-6}$ when compared with GluRIIIA ${ }^{\text {sp16 }}$. However, GluRIIA $A^{\text {sp } 16}$; $\mathrm{wg}^{1-12} /+; g b^{01155} /+$ animals have restored homeostasis (mEPSP $=0.34 \pm 0.02 \mathrm{mV}$; $\mathrm{EPSP}=26.8 \pm 2.4 \mathrm{mV}$; quantal content $=81.2 \pm 8.5 ; n=14) ; \mathrm{QC}$ is increased by $79 \pm$ $18.8 \%$ compared with control values. This is significantly different from GluRIIIAp16; $g 5 b^{01155} /+$ animals $(p=0.02)$ but not different from GluRIIIA $A^{\text {sp16 }}(p=0.85)$ or GluRIIA $A^{5 p 16} ; \mathrm{Wg} /+(p=$ $0.83)$. Error bars represent average \pm SEM.

the GluRIIA mutation represents a persistent stress that induces a continuous, rapidly induced form of homeostatic compensation. In this model, the homeostatic modulation of presynaptic release is continually updated and never consolidated. An alternative model is that, once induced, the homeostatic modulation of presynaptic release is consolidated and maintained for prolonged periods of time. If this is the case, it should be possible to selectively disrupt the consolidation of synaptic homeostasis independently of the mechanisms of induction. Loss of Gsb appears to do just this. It is not possible to persistently inhibit protein synthesis during larval development. However, the demonstration that decreased Gsb disrupts synaptic homeostasis in the GluRIIA mutant suggests that transcription and translation may be involved in the mechanisms that consolidate a homeostatic change in presynaptic release.

Gsb selectively impairs the mechanisms responsible for a homeostatic increase in presynaptic release

Several studies have now demonstrated that synaptic homeostasis is bidirectional at the Drosophila NMJ, being able to increase or decrease presynaptic vesicle release (Davis and Goodman, 1998; Daniels et al., 2004). It is currently unknown whether the same molecular mechanisms are responsible for the observed homeostatic increase versus decrease in presynaptic release. Here, we demonstrate that loss of Gsb disrupts the homeostatic increase of presynaptic release observed in the GluRIIA mutant background, but it does not alter the homeostatic decrease in presynaptic release observed when the vGlut transporter is driven presynaptically and quantal size is thereby increased. These data suggest that different molecular mechanisms could be involved in the consolidation of a homeostatic increase in release versus a homeostatic decrease in presynaptic release. The possibility that Gsb participates only in the expression of a homeostatic increase in release may suggest something about the role of Gsb within this homeostatic signaling system. It is hypothesized, at the Drosophila NMJ, that altered postsynaptic excitation, either an increase or a decrease in glutamate receptor activation, is somehow detected by a monitor that resides within the postsynaptic muscle cell (Davis, 2006). Similar monitors of cellular excitation have been invoked in other neural systems, including central neurons, and evidence has been presented that altered calcium signaling participates in the monitoring of cellular excitability (Liu et al., 1998; Turrigiano and Nelson, 2000; Marder and Prinz, 2002; PiedrasRentería et al., 2004; Thiagarajan et al., 2005). Since loss of Gsb does not impair the ability of the neuromuscular system to homeostatically adapt to an increase in quantal size, and since the rapid induction of homeostatic signaling is intact, it is suggested that the postsynaptic monitor of muscle glutamate receptor activation is unaffected by the loss of Gsb. This possibility is consistent with the observation that Gsb is expressed only in the presynaptic neuron. Thus, Gsb appears to function downstream of the "homeostatic sensor" and is somehow involved in the mechanisms that sustain a homeostatic increase in presynaptic release over time.

\section{Homeodomain transcription factors and evidence that} $w g$ functions as an antagonist of synaptic homeostasis

In Drosophila, like in vertebrates, combinations of transcriptional regulators determine the fate of neurons (for review, see Edlund and Jessell, 1999; Jessell, 2000; Shirasaki and Pfaff, 2002). Indeed, transcription factors control all stages of early neuronal development and neuronal circuit formation, from the direction in which the axon initially extends from the neuronal cell body (Landgraf et al., 1999; Thor et al., 1999; Kania et al., 2000), the location of the terminal zone of the axonal arborization (Arber et al., 2000), and the specificity of synaptic targeting (Landgraf and Thor, 2006) to the choice of neurotransmitter (Bröhl et al., 2008). 
More recently, some evidence suggests that expression of the transcription factor evenskipped during early embryogenesis could affect a motoneuron's complement of ion channels and neuron excitability (Pym et al., 2006). However very little is known regarding the role of transcriptional regulators in mature neurons (Mee et al., 2004). Recently, it was demonstrated that mild perturbation of the engrailed gene lead to mice with an adult phenotype that resembles key pathological features of Parkinson's disease (Sgadò et al., 2006; Sonnier et al., 2007). In our study, the expression of Gsb-RNAi using a postmitotic neuronal GAL4 driver allows us to conclude that Gsb has a postmitotic activity that is essential to the maintenance of synaptic homeostasis. These data provide evidence that the transcription factors involved in embryonic development may have potent postembryonic functions that are necessary for the maintenance of stable neural function.

How do embryonic transcriptional regulators influence the expression of synaptic homeostasis? We present data to support a model in which Gsb function is conserved during embryonic patterning (Duman-Scheel et al., 1997; Bhat et al., 2000) and synaptic homeostasis (Fig. $10 \mathrm{~A}$ ). Specifically, decreased $w g$ levels rescue synaptic homeostasis in GluRIIA; $g s b /+$ double mutant animals. According to this model, Wg antagonizes the expression or consolidation of synaptic homeostasis, providing new insight into the activity of this potent intercellular, synaptic signaling molecule. It could be important, for example, to suppress homeostatic signaling during anatomical synaptic plasticity, a process in which Wg has been implicated (Ataman et al., 2008). These data are strengthened by two observations. First, these data are supported by the well established embryonic activity of Gsb (Duman-Scheel et al., 1997; Bhat et al., 2000). Second, since partial loss of $w g$ rescues the homeostatic defect in GluRIIA; gsb/+ double mutant animals, it is unlikely that this represents a nonspecific genetic interaction. Many additional experiments will be necessary to prove the function of $w g$ as an antagonist of synaptic homeostasis. Our data, however, take this model beyond the stage of pure speculation and suggest that this will be an important avenue of future experimental investigation.

\section{References}

Arber S, Ladle DR, Lin JH, Frank E, Jessell TM (2000) ETS gene Er81 controls the formation of functional connections between group Ia sensory afferents and motor neurons. Cell 101:485-498.

Ataman B, Ashley J, Gorczyca M, Ramachandran P, Fouquet W, Sigrist SJ, Budnik V (2008) Rapid activity-dependent modifications in synaptic structure and function require bidirectional Wnt signaling. Neuron 57:705-718.

Baldwin CT, Hoth CF, Amos JA, da-Silva EO, Milunsky A (1992) An exonic mutation in the HuP2 paired domain gene causes Waardenburg's syndrome. Nature 355:637-638.

Bernard C, Anderson A, Becker A, Poolos NP, Beck H, Johnston D (2004) Acquired dendritic channelopathy in temporal lobe epilepsy. Science 305:532-535.

Bhat KM, van Beers EH, Bhat P (2000) Sloppy paired acts as the downstream target of Wingless in the Drosophila CNS and interaction between sloppy paired and gooseberry inhibits sloppy paired during neurogenesis. Development 127:655-665.

Brand AH, Perrimon N (1993) Targeted gene expression as a means of altering cell fates and generating dominant phenotypes. Development 118:401-415.

Bröhl D, Strehle M, Wende H, Hori K, Bormuth I, Nave KA, Müller T, Birchmeier C (2008) A transcriptional network coordinately determines transmitter and peptidergic fate in the dorsal spinal cord. Dev Biol 322:381-393

Budnik V, Koh YH, Guan B, Hartmann B, Hough C, Woods D, Gorczyca M (1996) Regulation of synapse structure and function by the Drosophila tumor suppressor gene dlg. Neuron 17:627-640.
Charron F, Tessier-Lavigne M (2005) Novel brain wiring functions for classical morphogens: a role as graded positional cues in axon guidance. Development 132:2251-2262.

Couso JP, Martinez Arias A (1994) Notch is required for wingless signaling in the epidermis of Drosophila. Cell 79:259-272.

Dahl E, Koseki H, Balling R (1997) Pax genes and organogenesis. Bioessays 19:755-765.

Daniels RW, Collins CA, Gelfand MV, Dant J, Brooks ES, Krantz DE, DiAntonio A (2004) Increased expression of the Drosophila vesicular glutamate transporter leads to excess glutamate release and a compensatory decrease in quantal content. J Neurosci 24:10466-10474.

Davis EK, Zou Y, Ghosh A (2008) Wnts acting through canonical and noncanonical signaling pathways exert opposite effects on hippocampal synapse formation. Neural Dev 3:32.

Davis GW (2006) Homeostatic control of neural activity: from phenomenology to molecular design. Annu Rev Neurosci 29:307-323.

Davis GW, Goodman CS (1998) Genetic analysis of synaptic development and plasticity: homeostatic regulation of synaptic efficacy. Curr Opin Neurobiol 8:149-156.

Davis GW, DiAntonio A, Petersen SA, Goodman CS (1998) Postsynaptic PKA controls quantal size and reveals a retrograde signal that regulates presynaptic transmitter release in Drosophila. Neuron 20:305-315.

Deshpande N, Dittrich R, Technau GM, Urban J (2001) Successive specification of Drosophila neuroblasts NB 6-4 and NB 7-3 depends on interaction of the segment polarity genes wingless, gooseberry and naked cuticle. Development 128:3253-3261.

DiAntonio A, Petersen SA, Heckmann M, Goodman CS (1999) Glutamate receptor expression regulates quantal size and quantal content at the Drosophila neuromuscular junction. J Neurosci 19:3023-3032.

Duman-Scheel M, Li X, Orlov I, Noll M, Patel NH (1997) Genetic separation of the neural and cuticular patterning functions of gooseberry. Development 124:2855-2865.

Edlund T, Jessell TM (1999) Progression from extrinsic to intrinsic signaling in cell fate specification: a view from the nervous system. Cell 96:211-224.

Frank CA, Kennedy MJ, Goold CP, Marek KW, Davis GW (2006) Mechanisms underlying the rapid induction and sustained expression of synaptic homeostasis. Neuron 52:663-667.

Frank CA, Pielage J, Davis GW (2009) A presynaptic homeostatic signaling system composed of the Eph receptor ephexin, Cdc42, and CaV2.1 calcium channels. Neuron 61:556-569.

Fdez E, Hilfiker S (2006) Vesicle pools and synapsins: new insights into old enigmas. Brain Cell Biol 35:107-115.

Gogolla N, Galimberti I, Deguchi Y, Caroni P (2009) Wnt signaling mediates experience-related regulation of synapse numbers and mossy fiber connectivities in the adult hippocampus. Neuron 62:510-525.

Goold CP, Davis GW (2007) The BMP ligand Gbb gates the expression of synaptic homeostasis independent of synaptic growth control. Neuron 56:109-123.

Gutjahr T, Patel NH, Li X, Goodman CS, Noll M (1993) Analysis of the gooseberry locus in Drosophila embryos: gooseberry determines the cuticular pattern and activates gooseberry neuro. Development 118:21-31.

Henriquez JP, Webb A, Bence M, Bildsoe H, Sahores M, Hughes SM, Salinas PC (2008) Wnt signaling promotes AChR aggregation at the neuromuscular synapse in collaboration with agrin. Proc Natl Acad Sci U S A 105:18812-18817.

Hough CD, Woods DF, Park S, Bryant PJ (1997) Organizing a functional junctional complex requires specific domains of the Drosophila MAGUK Discs large. Genes Dev 11:3242-3253.

Houweling AR, Bazhenov M, Timofeev I, Steriade M, Sejnowski TJ (2005) Homeostatic synaptic plasticity can explain posttraumatic epileptogenesis in chronically isolated neocortex. Cereb Cortex 15:834-845.

Jakubs K, Nanobashvili A, Bonde S, Ekdahl CT, Kokaia Z, Kokaia M, Lindvall O (2006) Environment matters: synaptic properties of neurons born in the epileptic adult brain develop to reduce excitability. Neuron 52:1047-1059.

Jessell TM (2000) Neuronal specification in the spinal cord: inductive signals and transcriptional codes. Nat Rev Genet 1:20-29.

Jing L, Lefebvre JL, Gordon LR, Granato M (2009) Wnt signals organize synaptic prepattern and axon guidance through the zebrafish unplugged/ MuSK receptor. Neuron 61:721-733.

Kania A, Johnson RL, and Jessell TM (2000) Coordinate roles for LIM ho- 
meobox genes in directing the dorsoventral trajectory of motor axons in the vertebrate limb. Cell 102:161-173.

Kittel RJ, Wichmann C, Rasse TM, Fouquet W, Schmidt M, Schmid A, Wagh DA, Pawlu C, Kellner RR, Willig KI, Hell SW, Buchner E, Heckmann M, Sigrist SJ (2006) Bruchpilot promotes active zone assembly, $\mathrm{Ca}^{2+}$ channel clustering, and vesicle release. Science 312:1051-1054.

Klagges BR, Heimbeck G, Godenschwege TA, Hofbauer A, Pflugfelder GO, Reifegerste R, Reisch D, Schaupp M, Buchner S, Buchner E (1996) Invertebrate synapsins: a single gene codes for several isoforms in Drosophila. J Neurosci 16:3154-3165.

Korkut C, Budnik V (2009) WNTs tune up the neuromuscular junction. Nat Rev Neurosci 10:627-634.

Korkut C, Ataman B, Ramachandran P, Ashley J, Barria R, Gherbesi N, Budnik V (2009) Trans-synaptic transmission of vesicular Wnt signals through Evi/ Wntless. Cell 139:393-404.

Landgraf M, Thor S (2006) Development of Drosophila motoneurons: specification and morphology. Semin Cell Dev Biol 17:3-11.

Landgraf M, Roy S, Prokop A, VijayRaghavan K, Bate M (1999) evenskipped determines the dorsal growth of motor axons in Drosophila. Neuron 22:43-52.

Lee YS, Carthew RW (2003) Making a better RNAi vector for Drosophila: use of intron spacers. Methods 30:322-329.

Li X, Noll M (1993) Role of the gooseberry gene in Drosophila embryos: maintenance of wingless expression by a wingless-gooseberry autoregulatory loop. EMBO J 12:4499-4509.

Liu Z, Golowasch J, Marder E, Abbott LF (1998) A model neuron with activity-dependent conductances regulated by multiple calcium sensors. J Neurosci 18:2309-2320.

Marder E, Goaillard JM (2006) Variability, compensation and homeostasis in neuron and network function. Nat Rev Neurosci 7:563-574.

Marder E, Prinz AA (2002) Modeling stability in neuron and network function: the role of activity in homeostasis. Bioessays 24:1145-1154.

Marie B, Sweeney ST, Poskanzer KE, Roos J, Kelly RB, Davis GW (2004) Dap160/intersectin scaffolds the periactive zone to achieve high-fidelity endocytosis and normal synaptic growth. Neuron 43:207-219.

Mathew D, Ataman B, Chen J, Zhang Y, Cumberledge S, Budnik V (2005) Wingless signaling at synapses is through cleavage and nuclear import of receptor DFrizzled2. Science 310:1344-1347.

Mee CJ, Pym EC, Moffat KG, Baines RA (2004) Regulation of neuronal excitability through pumilio-dependent control of a sodium channel gene. J Neurosci 24:8695-8703.

Miech C, Pauer HU, He X, Schwarz TL (2008) Presynaptic local signaling by a canonical wingless pathway regulates development of the Drosophila neuromuscular junction. J Neurosci 28:10875-10884.

Packard M, Koo ES, Gorczyca M, Sharpe J, Cumberledge S, Budnik V (2002) The Drosophila Wnt, wingless, provides an essential signal for pre- and postsynaptic differentiation. Cell 111:319-330.

Petersen SA, Fetter RD, Noordermeer JN, Goodman CS, DiAntonio A (1997) Genetic analysis of glutamate receptors in Drosophila reveals a retrograde signal regulating presynaptic transmitter release. Neuron 19:1237-1248.

Piedras-Rentería ES, Pyle JL, Diehn M, Glickfeld LL, Harata NC, Cao Y, Kavalali ET, Brown PO, Tsien RW (2004) Presynaptic homeostasis at CNS nerve terminals compensates for lack of a key $\mathrm{Ca}^{2+}$ entry pathway. Proc Natl Acad Sci U S A 101:3609-3614.

Pielage J, Fetter RD, Davis GW (2005) Presynaptic spectrin is essential for synapse stabilization. Curr Biol 15:918-928.

Pielage J, Fetter RD, Davis GW (2006) A postsynaptic spectrin scaffold defines active zone size, spacing, and efficacy at the Drosophila neuromuscular junction. J Cell Biol 175:491-503.

Pielage J, Cheng L, Fetter RD, Carlton PM, Sedat JW, Davis GW (2008) A pre- synaptic giant ankyrin stabilizes the NMJ through regulation of presynaptic microtubules and transsynaptic cell adhesion. Neuron 58:195-209.

Poon VY, Klassen MP, Shen K (2008) UNC-6/netrin and its receptor UNC-5 locally exclude presynaptic components from dendrites. Nature 455:669-673.

Pym EC, Southall TD, Mee CJ, Brand AH, Baines RA (2006) The homeobox transcription factor Even-skipped regulates acquisition of electrical properties in Drosophila neurons. Neural Develop 1:3.

Rohrbough J, Broadie K (2002) Electrophysiological analysis of synaptic transmission in central neurons of Drosophila larvae. J Neurophysiol 88:847-860.

Roos J, Kelly RB (1998) Dap160, a neural-specific Eps15 homology and multiple SH3 domain-containing protein that interacts with Drosophila dynamin. J Biol Chem 273:19108-19119.

Sever S (2002) Dynamin and endocytosis. Curr Opin Cell Biol 14:463-467.

Sgadò P, Albéri L, Gherbassi D, Galasso SL, Ramakers GM, Alavian KN, Smidt MP, Dyck RH, Simon HH (2006) Slow progressive degeneration of nigral dopaminergic neurons in postnatal Engrailed mutant mice. Proc Natl Acad Sci U S A 103:15242-15247.

Shirasaki R, Pfaff SL (2002) Transcriptional codes and the control of neuronal identity. Annu Rev Neurosci 25:251-281.

Skeath JB, Zhang Y, Holmgren R, Carroll SB, Doe CQ (1995) Specification of neuroblast identity in the Drosophila embryonic central nervous system by gooseberry-distal. Nature 376:427-430.

Sonnier L, Le Pen G, Hartmann A, Bizot JC, Trovero F, Krebs MO, Prochiantz A (2007) Progressive loss of dopaminergic neurons in the ventral midbrain of adult mice heterozygote for Engrailed1. J Neurosci 27:1063-1071.

Tassabehji M, Read AP, Newton VE, Harris R, Balling R, Gruss P, Strachan T (1992) Waardenburg's syndrome patients have mutations in the human homologue of the Pax-3 paired box gene. Nature 355:635-636.

Tassabehji M, Read AP, Newton VE, Patton M, Gruss P, Harris R, Strachan T (1993) Mutations in the PAX3 gene causing Waardenburg syndrome type 1 and type 2. Nat Genet 3:26-30.

Thiagarajan TC, Lindskog M, Tsien RW (2005) Adaptation to synaptic inactivity in hippocampal neurons. Neuron 47:725-737.

Thor S, Andersson SG, Tomlinson A, Thomas JB (1999) A LIM-homeodomain combinatorial code for motor-neuron pathway selection. Nature 397:76-80.

Turrigiano GG (2008) The self-tuning neuron: synaptic scaling of excitatory synapses. Cell 135:422-435.

Turrigiano GG, Nelson SB (2000) Hebb and homeostasis in neuronal plasticity. Curr Opin Neurobiol 10:358-364. Review.

van den Heuvel M, Harryman-Samos C, Klingensmith J, Perrimon N, Nusse R (1993) Mutations in the segment polarity genes wingless and porcupine impair secretion of the wingless protein. EMBO J 12:5293-5302.

Waardenburg P (1951) A new syndrome combining developmental anomalies of the eyelids, eyebrows, and nose root with congenital deafness. Am J Hum Genet 3:195-253.

Wagh DA, Rasse TM, Asan E, Hofbauer A, Schwenkert I, Dürrbeck H, Buchner S, Dabauvalle MC, Schmidt M, Qin G, Wichmann C, Kittel R, Sigrist SJ, Buchner E (2006) Bruchpilot, a protein with homology to ELKS/CAST, is required for structural integrity and function of synaptic active zones in Drosophila. Neuron 49:833-844. Erratum in: Neuron 51:27.

Wehr R, Gruss P (1996) Pax and vertebrate development. Int J Dev Biol 40:369-377.

Wucherpfennig T, Wilsch-Bräuninger M, González-Gaitán M (2003) Role of Drosophila Rab5 during endosomal trafficking at the synapse and evoked neurotransmitter release. J Cell Biol 161:609-624.

Zhang Y, Ungar A, Fresquez C, Holmgren R (1994) Ectopic expression of either the Drosophila gooseberry-distal or proximal gene causes alterations of cell fate in the epidermis and central nervous system. Development 120:1151-1161. 